\title{
First steps in brave new commutative algebra.
}

\author{
J.P.C. Greenlees
}

\section{Contents}

1. Introduction 1

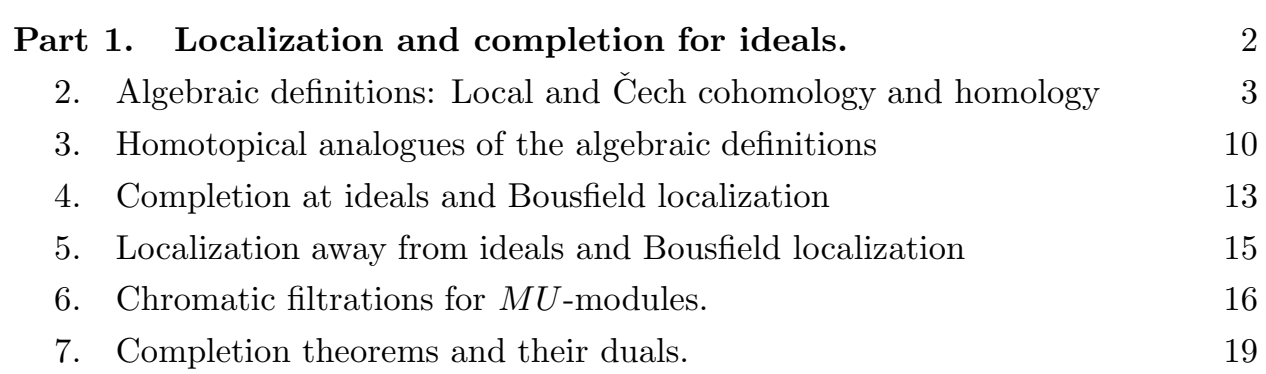

Part 2. Morita equivalences and Gorenstein rings. 21

8. The context. and some examples. 21

9. Morita equivalences. 24

10. Matlis lifts. 28

11. The Gorenstein condition. 31

$\begin{array}{ll}\text { References } & 35\end{array}$

\section{Introduction}

To begin with, it is helpful to explain the title. The phrase 'brave new rings' was coined by F.Waldhausen, presumably to capture both an optimism about the possibilities of generalizing rings to ring spectra, and a proper awareness of the risk that the new step in abstraction would take the subject dangerously far from

1991 Mathematics Subject Classification. Primary 55P43; Secondary 13D25, 13D45, 55P91, $55 \mathrm{U} 99$.

(C) 0000 (copyright holder) 
its justification in examples. This article is about doing commutative algebra with ring spectra, and is extensively illustrated by examples. The 'First steps' of the title suggests its design is controlled by expository imperatives, but in fact the first steps also follow one particular line of historical development. From either point of view, this gives a coherent story.

From the expository point of view, we could explain the choice as follows: completion and localization are very basic constructions in commutative algebra, and their formal nature makes them natural things to investigate in derived categories. Part 1 describes a naive approach through elements, and Part 2 moves on to a more conceptual approach, both illustrated by examples.

From the historical point of view, one route begins with the Atiyah-Segal completion theorem about the $K$-theory of classifying spaces $[\mathbf{3}$. For a finite group $G$ this is the statement that

$$
K^{0}(B G)=K_{G}^{0}(E G)=K_{G}^{0}(p t)_{J}^{\wedge}=R(G)_{J}^{\wedge}
$$

where $R(G)$ is the complex representation ring and $J$ is its augmentation ideal. It is natural to try to lift the algebraic statements to structural statements at the level of derived categories of spectra. In this context, the philosophy is that the theorem states that an algebraic and a geometric completion agree. The algebra this uncovers then suggests a new approach to the Atiyah-Segal completion theorem 27, by starting with a version for homology. It turns out that this phenomenon occurs in many contexts and in some of the more algebraic ones the role of Morita theory begins to emerge. This leads on to a more conceptual approach, which, like Gorenstein rings [6], turns out to occur ubiquitously.

This article is based on the lectures I gave at the meeting in Chicago in Summer 2004. The points of view described have developed in joint work with Benson, Dwyer, Iyengar and May [19, 9, 34, 35; I am grateful to them all for numerous conversations and insights.

\section{Part 1. Localization and completion for ideals.}

Localization and completion are basic constructions in commutative algebra, and it is useful to be able to use them in stable homotopy theory. However, they can be viewed as just one special case of categorically similar constructions. Several important theorems can then be viewed as change of base results in this broadened context.

We begin with constructions in commutative algebra, and work towards formulating them in a way which generalizes to other derived categories. Consider

an ideal $I$ in a commutative ring $R$, and then attempt to approximate modules by 
I-torsion modules. If we use inverse limits, we reach the notion of the completion

$$
M_{I}^{\wedge}=\lim _{\leftarrow k} M / I^{k} M
$$

of an $R$-module $M$. The algebraic fact that completion is not exact in the nonNoetherian case means that in treating homological invariants, we are forced to work with the derived functors of completion. In fact topology suggests a homotopy invariant construction which leads in turn to a means of calculation of the left derived functors of completion (local homology); this then models the the completion of spectra by construction.

We began by considering inverse limits of torsion modules since completion is exact on Noetherian modules, so in that case the zeroth derived functor agrees with the original and the higher derived functors are zero. On the other hand, if we attempt to approximate modules by direct limits of torsion modules we obtain

$$
\Gamma_{I} M=\lim _{\rightarrow k} \operatorname{Hom}_{R}\left(A / I^{k}, M\right)=\left\{x \mid I^{k} x=0 \text { for } k>>0\right\} .
$$

This time the original functor is often zero (in the torsion free case for example), and it is the higher derived functors that are better behaved. These are calculated by a dual construction, which leads to Grothendieck's local cohomology modules.

We will begin in Section 2 with the familiar commutative algebra, and then adapt it to ring spectra and study it in Sections 3 to 5 Finally we consider two specializations: that to $M U$-module spectra in Section [6] when we obtain well known chromatic constructions, and the motivating examples in equivariant topology in Section $\mathbf{7}$ Related surveys are given in [36, 37.

\section{Algebraic definitions: Local and Čech cohomology and homology}

The material in this section is based on $[41,35,28$. Background in commutative algebra can be found in $48, \mathbf{1 3}$.

2.A. The functors. Suppose to begin with that $R$ is a commutative Noetherian ring and that $I=\left(\alpha_{1}, \ldots, \alpha_{n}\right)$ is an ideal in $R$. We shall be concerned especially with two naturally occurring functors on $R$-modules: the $I$-power torsion functor and the $I$-adic completion functor.

The $I$-power torsion functor $\Gamma_{I}$ is defined by

$$
M \longmapsto \Gamma_{I}(M)=\left\{x \in M \mid I^{k} x=0 \text { for } k>>0\right\} .
$$

We say that $M$ is an $I$-power torsion module if $M=\Gamma_{I} M$. It is easy to check that the functor $\Gamma_{I}$ is left exact.

Recall that the support of $M$ is the set of prime ideals $\wp$ of $R$ such that the localization $M_{\wp}$ is non-zero. We say that $M$ is supported over $I$ if every prime in the support of $M$ contains $I$. This is equivalent to the condition that $M[1 / \alpha]=0$ 
for each $\alpha \in I$. It follows that $M$ is an $I$-power torsion module if and only if the support of $M$ lies over $I$.

The $I$-adic completion functor is defined by

$$
M \longmapsto M_{I}^{\wedge}=\lim _{\leftarrow k} M / I^{k} M
$$

The Artin-Rees lemma implies that $I$-adic completion is exact on finitely generated modules, but it is neither right nor left exact in general.

Since the functors that arise in topology are exact functors on triangulated categories, we need to understand the algebraic functors at the level of the derived category, which is to say that we must understand their derived functors. The connection with topology comes through one particular way of calculating the derived functors $R^{*} \Gamma_{I}$ of $\Gamma_{I}$ and $L_{*}^{I}$ of $I$-adic completion. It also provides a connection between the two sets of derived functors and makes useful techniques available.

2.B. The stable Koszul complex. We begin with a sequence $\alpha_{1}, \ldots, \alpha_{n}$ of elements of $R$ and define various chain complexes. In Subsection 2.C we explain why the chain complexes only depend on the radical of the ideal $I=\left(\alpha_{1}, \ldots, \alpha_{n}\right)$ generated by the sequence, in Subsection 2.D we define associated homology groups, and in Subsection 2.E we give conceptual interpretations of this homology under Noetherian hypotheses.

We begin with a single element $\alpha \in R$, and an integer $s \geq 0$, and define the sth unstable Koszul complex by

$$
K_{s}^{\bullet}(\alpha)=\left(\alpha^{s}: R \longrightarrow R\right)
$$

where the non-zero modules are in cohomological degrees 0 and 1 . These complexes form a direct system as $s$ varies,

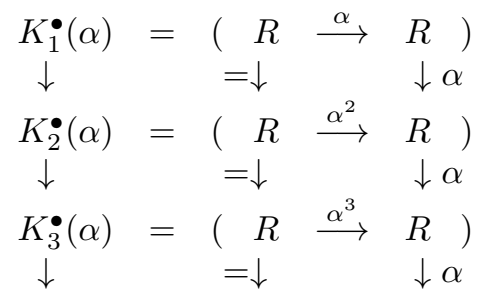

and the direct limit is the flat stable Koszul complex

$$
K^{\bullet}(\alpha)=(R \longrightarrow R[1 / \alpha]) .
$$

When defining local cohomology, it is usual to use the complex $K^{\bullet}(\alpha)$ of flat modules. However, we shall need a complex of projective $R$-modules to define the dual local homology modules. Accordingly, we take a particularly convenient projective approximation $P K^{\bullet}(\alpha)$ to $K^{\bullet}(\alpha)$. Instead of taking the direct limit of the $K_{s}^{\bullet}(\alpha)$, we take their homotopy direct limit. This makes the translation to 
the topological context straightforward. More concretely, our model for $P K^{\bullet}(\alpha)$ is displayed as the upper row in the homology isomorphism

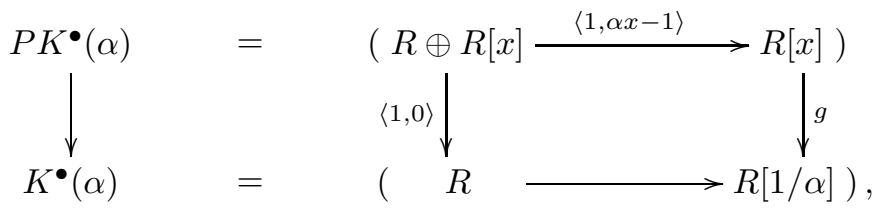

where $g\left(x^{i}\right)=1 / \alpha^{i}$. Like $K^{\bullet}(\alpha)$, this choice of $P K^{\bullet}(\alpha)$ is non-zero only in cohomological degrees 0 and 1 .

The stable Koszul cochain complex for a sequence $\boldsymbol{\alpha}=\left(\alpha_{1}, \ldots, \alpha_{n}\right)$ is obtained by tensoring together the complexes for the elements, so that

$$
K^{\bullet}(\boldsymbol{\alpha})=K^{\bullet}\left(\alpha_{1}\right) \otimes_{R} \cdots \otimes_{R} K^{\bullet}\left(\alpha_{n}\right),
$$

and similarly for the projective complex $P K^{\bullet}(\boldsymbol{\alpha})$.

2.C. Invariance statements. We prove some basic properties of the stable Koszul complex.

Lemma 2.1. If $\beta$ is in the ideal $I=\left(\alpha_{1}, \alpha_{2}, \ldots, \alpha_{n}\right)$, then $K^{\bullet}(\boldsymbol{\alpha})[1 / \beta]$ is exact.

Proof: Since homology commutes with direct limits, it suffices to show that some power of $\beta$ acts as zero on the homology of $K_{s}^{\bullet}(\boldsymbol{\alpha})=K_{s}^{\bullet}\left(\alpha_{1}\right) \otimes \cdots \otimes K_{s}^{\bullet}\left(\alpha_{n}\right)$. However, $\left(\alpha_{i}\right)^{s}$ annihilates $H^{*}\left(K_{s}^{\bullet}\left(\alpha_{i}\right)\right)$, and it follows from the long exact sequence in homology that $\left(\alpha_{i}\right)^{2 s}$ annihilates $H^{*}\left(K_{s}^{\bullet}(\boldsymbol{\alpha})\right)$. Writing $\beta$ as a linear combination of the $n$ elements $\alpha_{i}$, we see that $\beta^{2 s n}$ is a linear combination of elements each of which is divisible by some $\left(\alpha_{i}\right)^{2 s}$, and the conclusion follows.

Note that, by construction, we have an augmentation map

$$
\varepsilon: K^{\bullet}(\boldsymbol{\alpha}) \longrightarrow R .
$$

Corollary 2.2. Up to quasi-isomorphism, the complex $K^{\bullet}(\boldsymbol{\alpha})$ depends only on the radical of the ideal $I$.

Proof: Since $K^{\bullet}(\boldsymbol{\alpha})$ is unchanged if we replace the generators by powers, it suffices to show that $K^{\bullet}(\boldsymbol{\alpha})$ only depends on the ideal generated by $\boldsymbol{\alpha}$.

The augmentation gives a map

$$
K^{\bullet}(\boldsymbol{\alpha}, \beta)=K^{\bullet}(\boldsymbol{\alpha}) \otimes_{R} K^{\bullet}(\beta) \longrightarrow K^{\bullet}(\boldsymbol{\alpha}) \otimes_{R} R=K^{\bullet}(\boldsymbol{\alpha})
$$

which is a quasi-isomorphism if $\beta \in I$ since the lemma shows its cofibre $K^{\bullet}(\boldsymbol{\alpha})[1 / \beta]$ is exact. It follows that we have homology isomorphisms

$$
K^{\bullet}(\boldsymbol{\alpha}) \longleftarrow K^{\bullet}(\boldsymbol{\alpha}) \otimes K^{\bullet}\left(\boldsymbol{\alpha}^{\prime}\right) \longrightarrow K^{\bullet}\left(\boldsymbol{\alpha}^{\prime}\right)
$$

if $\boldsymbol{\alpha}^{\prime}$ is a second set of generators for $I$. 
In view of Corollary 2.2 it is reasonable to write $K^{\bullet}(I)$ for $K^{\bullet}(\boldsymbol{\alpha})$. Since $P K^{\bullet}(\boldsymbol{\alpha})$ is a projective approximation to $K^{\bullet}(\boldsymbol{\alpha})$, it too depends only on the radical of $I$. We also write $K_{s}^{\bullet}(I)=K_{s}^{\bullet}\left(\alpha_{1}\right) \otimes \cdots \otimes K_{s}^{\bullet}\left(\alpha_{n}\right)$, but this is an abuse of notation since even its homology groups do depend on the choice of generators.

2.D. Local homology and cohomology. The local cohomology and homology of an $R$-module $M$ are then defined by

$$
H_{I}^{*}(R ; M)=H^{*}\left(P K^{\bullet}(I) \otimes M\right)
$$

and

$$
H_{*}^{I}(R ; M)=H_{*}\left(\operatorname{Hom}\left(P K^{\bullet}(I), M\right) .\right.
$$

Note that we could equally well use the flat stable Koszul complex in the definition of local cohomology, as is more usual. Lemma 2.1] shows that $H_{I}^{*}(M)[1 / \beta]=0$ if $\beta \in I$, so $H_{I}^{*}(M)$ is an $I$-power torsion module and supported over $I$.

It is immediate from the definitions that local cohomology and local homology are related by a third quadrant universal coefficient spectral sequence

$$
E_{2}^{s, t}=\operatorname{Ext}_{R}^{s}\left(H_{I}^{-t}(R), M\right) \Longrightarrow H_{-t-s}^{I}(R ; M),
$$

with differentials $d_{r}: E_{r}^{s, t} \longrightarrow E_{r}^{s+r, t-r+1}$.

We observe that local cohomology and homology are invariant under change of base ring.

Lemma 2.3. If $R \longrightarrow R^{\prime}$ is a ring homomorphism, $I^{\prime}$ is the ideal $I \cdot R^{\prime}$ and $M^{\prime}$ is an $R^{\prime}$-module regarded by pullback as an $R$-module, then

$$
H_{I}^{*}\left(R ; M^{\prime}\right) \cong H_{I^{\prime}}^{*}\left(R^{\prime} ; M^{\prime}\right) \text { and } H_{*}^{I}\left(R ; M^{\prime}\right) \cong H_{*}^{I^{\prime}}\left(R^{\prime} ; M^{\prime}\right)
$$

Proof: The statement for local cohomology is immediate from the definition, since $R \otimes_{R} M^{\prime}=R^{\prime} \otimes_{R^{\prime}} M^{\prime}$. The statement for local homology is similar, for example from the explicit projective model for the stable Koszul complex, or it can be deduced from the local cohomology statement using the Universal Coefficient Theorem.

In view of this, we usually omit the ring $R$ from the notation unless is is necessary for emphasis.

It is also useful to know that local homology and cohomology are invariant under completion.

LEMMA 2.4. Local homology and cohomology are invariant under the completion $M \longrightarrow M_{I}^{\wedge}$ of a finitely generated module $M$. 
Proof: From the Universal Coefficient Theorem it suffices to prove the result for local cohomology.

First note that the kernel of the natural map $K^{\bullet}(\boldsymbol{\alpha}) \longrightarrow R$ has a finite filtration with subquotients $R[1 / \beta]$ for $\beta \in I$. Similarly, the kernel of the natural map $P K^{\bullet}(\boldsymbol{\alpha}) \longrightarrow R$ has a finite filtration with subquotients $P R[1 / \beta]$ for $\beta \in I$. Since $\beta$ is invertible on $\operatorname{Hom}_{R}(P R[1 / \beta], M)$, Lemma 2.1 shows $\operatorname{Hom}_{R}(P R[1 / \beta], M) \otimes_{R}$ $K^{\bullet}(\boldsymbol{\alpha})$ is contractible.

It follows that the natural map

$$
M \otimes_{R} K^{\bullet}(\boldsymbol{\alpha}) \stackrel{\simeq}{\longrightarrow} \operatorname{Hom}_{R}\left(P K^{\bullet}(\boldsymbol{\alpha}), M\right) \otimes_{R} K^{\bullet}(\boldsymbol{\alpha})
$$

is a homology isomorphism. We will see in 2.6 below that $\operatorname{Hom}_{R}\left(P K^{\bullet}(\boldsymbol{\alpha}), M\right) \simeq$ $M_{I}^{\wedge}$ for finitely generated modules $M$.

2.E. Derived functors. We gave our definitions in terms of specific chain complexes. The meaning of the definitions appears in the following two theorems.

Theorem 2.5 (Grothendieck [41]). If $R$ is Noetherian, then the local cohomology groups calculate the right derived functors of the left exact functor $M \longmapsto$ $\Gamma_{I}(M)$. In symbols,

$$
H_{I}^{n}(R ; M)=\left(R^{n} \Gamma_{I}\right)(M) .
$$

This result may be used to give an explicit expression for local cohomology in familiar terms. Indeed, since $\Gamma_{I}(M)=\lim _{\rightarrow r} \operatorname{Hom}\left(R / I^{r}, M\right)$, and the right derived functors of the right-hand side are obvious, we have

$$
\left(R^{n} \Gamma_{I}\right)(M) \cong \lim _{\rightarrow r} \operatorname{Ext}_{R}^{n}\left(R / I^{r}, M\right)
$$

The description in terms of the stable Koszul complex is usually more practical.

Theorem 2.6 (Greenlees-May [35]). If $R$ is Noetherian, then the local homology groups calculate the left derived functors of the (not usually right exact) $I$-adic completion functor $M \longmapsto M_{I}^{\wedge}$. Writing $L_{n}^{I}$ for the left derived functors of I-adic completion, this gives

$$
H_{n}^{I}(R ; M)=L_{n}^{I}(M)
$$

The conclusions of Theorem 2.5 and 2.6 are true under much weaker hypotheses [35, [52.

An elementary proof of Theorem [2.5] can be obtained by induction on the number of generators of $I$. To give the idea, we prove the principal case $I=(\alpha)$. It is clear that $H_{I}^{0}(Q)=\Gamma_{I}(Q)$ so it suffices to prove $H_{I}^{1}(Q)=\operatorname{cok}(Q \longrightarrow Q[1 / \alpha])=0$ when $Q$ is an injective $R$-module. 
Since $R$ is Noetherian, there is an $r$ for which $\alpha^{r+1} x=0$ implies $\alpha^{r} x=0$, and hence $R \longrightarrow(\alpha) \oplus R /\left(\alpha^{r}\right)$ is a monomorphism. By injectivity of $Q$, any map $q: R \longrightarrow Q$ extends to a map $\langle f, g\rangle:(\alpha) \oplus R /\left(\alpha^{r}\right) \longrightarrow Q$, and hence $q=f(\alpha)+g(1)$. On the other hand, again by injectivity of $Q$, the map $f:(\alpha) \longrightarrow Q$ extends to a map $f^{\prime}: R \longrightarrow Q$, so $f(\alpha)=\alpha f^{\prime}(1)$ is a multiple of $\alpha$, and we see $q=\alpha q^{\prime}+g(1)$ with $g(1)$ annihilated by $\alpha^{r}$. This shows

$$
Q=\alpha Q+\Gamma_{\alpha} Q
$$

so that $Q \longrightarrow Q[1 / \alpha]$ is an epimorphism as required.

The general case uses the spectral sequence

$$
H_{I}^{*}\left(H_{J}^{*}(M)\right) \Longrightarrow H_{I+J}^{*}(M)
$$

that is obtained from the isomorphism $P K^{\bullet}(I+J) \cong P K^{\bullet}(I) \otimes P K^{\bullet}(J)$. Since we have proved the principal case, the remaining step required for the general case (which we omit) is to show that if $Q$ is injective then $\Gamma_{I} Q$ is also injective.

The proof of Theorem 2.6 can also be obtained like this, although it is more complicated because the completion of a projective module is rarely projective.

2.F. The shape of local cohomology. One is used to the idea that $I$-adic completion is often exact, so that $L_{0}^{I}$ is the most significant of the left derived functors. However, it is the top non-vanishing right derived functor of $\Gamma_{I}$ that is the most significant. Some idea of the shape of these derived functors can be obtained from the following result. Observe that the complex $P K^{\bullet}(\boldsymbol{\alpha})$ is nonzero only in cohomological degrees between 0 and $n$, so that local homology and cohomology are zero above dimension $n$. A result of Grothendieck usually gives a much better bound. We write $\operatorname{dim}(R)$ for the Krull dimension of $R$ and $\operatorname{depth}_{I}(M)$ for the $I$-depth of a module $M$ (the length of the longest $M$-regular sequence from $I)$.

TheOREm 2.7 (Grothendieck [40]). If $R$ is Noetherian of Krull dimension $d$, then

$$
H_{I}^{i}(M)=0 \quad \text { and } \quad H_{i}^{I}(M)=0 \quad \text { if } i>d .
$$

If $e=\operatorname{depth}_{I}(M)$ then

$$
H_{I}^{i}(M)=0 \text { if } i<e .
$$

If $R$ is Noetherian, $M$ is finitely generated, and $I M \neq M$, then

$$
H_{I}^{e}(M) \neq 0 .
$$

Grothendieck's proof of vanishing begins by noting that local cohomology is sheaf cohomology with support. It then proceeds by induction on the Krull dimension and reduction to the irreducible case. The statement about depth is elementary, and proved by induction on the length of the $I$-sequence (see [48, 16.8]). 
The Universal Coefficient Theorem gives a useful consequence for local homology.

Corollary 2.8. If $R$ is Noetherian and $\operatorname{depth}_{I}(R)=\operatorname{dim}(R)=d$, then

$$
L_{s}^{I} M=\operatorname{Ext}_{R}^{d-s}\left(H_{I}^{d}(R), M\right) .
$$

For example if $R=\mathbb{Z}$ and $I=(p)$, then $H_{(p)}^{*}(\mathbb{Z})=H_{(p)}^{1}(\mathbb{Z})=\mathbb{Z} / p^{\infty}$. Therefore the corollary states that

$$
L_{0}^{(p)} M=\operatorname{Ext}\left(\mathbb{Z} / p^{\infty}, M\right) \quad \text { and } \quad L_{1}^{(p)} M=\operatorname{Hom}\left(\mathbb{Z} / p^{\infty}, M\right),
$$

as was observed in Bousfield-Kan [12, VI.2.1].

2.G. Čech homology and cohomology. We have motivated local cohomology in terms of $I$-power torsion, and it is natural to consider the difference between the torsion and the original module. In geometry this difference would be more fundamental than the torsion itself, and local cohomology would then arise by considering functions with support.

To construct a good model for this difference, observe that $\varepsilon: K^{\bullet}(\boldsymbol{\alpha}) \longrightarrow R$ is an isomorphism in degree zero and define the flat Čech complex $\check{C}^{\bullet}(I)$ to be the complex $\Sigma(\operatorname{ker} \varepsilon)$. Thus, if $i \geq 0$, then $\check{C}^{i}(I)=K^{i+1}(I)$. For example, if $I=(\alpha, \beta)$, then

$$
\check{C}^{\bullet}(I)=(R[1 / \alpha] \oplus R[1 / \beta] \longrightarrow R[1 /(\alpha \beta)]) .
$$

The differential $K^{0}(I) \longrightarrow K^{1}(I)$ specifies a chain map $R \longrightarrow \check{C}^{\bullet}(I)$ whose fibre is exactly $K^{\bullet}(I)$. Thus we have a fibre sequence

$$
K^{\bullet}(I) \longrightarrow R \longrightarrow \check{C}^{\bullet}(I) .
$$

We define the projective version $P C^{\bullet}(I)$ similarly, using the kernel of the composite of $\varepsilon$ and the quasi-isomorphism $P K^{\bullet}(I) \longrightarrow K^{\bullet}(I)$; note that $P \check{C}^{\bullet}(I)$ is non-zero in cohomological degree -1 .

The Čech cohomology and homology of an $R$-module $M$ are then defined by

$$
\check{C} H_{I}^{*}(R ; M)=H^{*}\left(P \check{C}^{\bullet}(I) \otimes M\right)
$$

and

$$
\check{C} H_{*}^{I}(R ; M)=H_{*}\left(\operatorname{Hom}\left(P \check{C}^{\bullet}(I), M\right)\right) .
$$

The Čech cohomology can also be defined by use of the flat Čech complex and is zero in negative degrees, but the Cech homology is usually non-zero in degree -1 .

The fibre sequence $P K^{\bullet}(I) \longrightarrow R \longrightarrow P \check{C}^{\bullet}(I)$ gives rise to long exact sequences relating local and Čech homology and cohomology,

$$
0 \longrightarrow H_{I}^{0}(M) \longrightarrow M \longrightarrow \check{C} H_{I}^{0}(M) \longrightarrow H_{I}^{1}(M) \longrightarrow 0
$$


and

$$
0 \longrightarrow H_{1}^{I}(M) \longrightarrow \check{C} H_{0}^{I}(M) \longrightarrow M \longrightarrow H_{0}^{I}(M) \longrightarrow \check{C} H_{-1}^{I}(M) \longrightarrow 0,
$$

together with isomorphisms

$$
H_{I}^{i}(M) \cong \check{C} H_{I}^{i-1}(M) \text { and } H_{i}^{I}(M) \cong \check{C} H_{i-1}^{I}(M) \text { for } i \geq 2 \text {. }
$$

2.H. Čech cohomology and Čech covers. To explain why $\check{C} \bullet(I)$ is called the Čech complex, we describe how it arises by using the Čech construction to calculate cohomology from a suitable open cover. More precisely, let $Y$ be the closed subscheme of $X=\operatorname{Spec}(R)$ determined by $I$. The space $V(I)=\{\wp \mid \wp \supset$ $I\}$ decomposes as $V(I)=V\left(\alpha_{1}\right) \cap \ldots \cap V\left(\alpha_{n}\right)$, and there results an open cover of the open subscheme $X-Y$ as the union of the complements $X-Y_{i}$ of the closed subschemes $Y_{i}$ determined by the principal ideals $\left(\alpha_{i}\right)$. However, $X-Y_{i}$ is isomorphic to the affine scheme $\operatorname{Spec}\left(R\left[1 / \alpha_{i}\right]\right)$. Since affine schemes have no higher cohomology,

$$
H^{*}\left(\operatorname{Spec}\left(R\left[1 / \alpha_{i}\right]\right) ; \tilde{M}\right)=H^{0}\left(\operatorname{Spec}\left(R\left[1 / \alpha_{i}\right]\right) ; \tilde{M}\right)=M\left[1 / \alpha_{i}\right],
$$

where $\tilde{M}$ is the sheaf associated to the $R$-module $M$. Thus the $E_{1}$ term of the Mayer-Vietoris spectral sequence for this cover collapses to the chain complex $\check{C}^{\bullet}(I)$, and

$$
H^{*}(X-Y ; \tilde{M}) \cong \check{C} H_{I}^{*}(M)
$$

\section{Homotopical analogues of the algebraic definitions}

We now transpose the algebra from Section 2 into the homotopy theoretic context. It is convenient to note that it is routine to extend the algebra to graded rings, and we will use this without further comment below. We assume the reader is already comfortable working with ring spectra, but there is an introduction with full references in $\mathbf{3 2}$, elsewhere in this volume.

We replace the standing assumption that $R$ is a commutative $\mathbb{Z}$-algebra by the assumption that it is a commutative $\mathbb{S}$-algebra, where $\mathbb{S}$ is the sphere spectrum. The category of $R$-modules is now the category of $R$-module spectra. Since the derived category of a ring $R$ is equivalent to the derived category of the associated Eilenberg-MacLane spectrum [54, the work of earlier sections can be reinterpreted in the new context. To emphasize the algebraic analogy, we write $\otimes_{R}$ and $\operatorname{Hom}_{R}$ for the smash product over $R$ and function spectrum of $R$-maps and 0 for the trivial module. In particular $X \otimes_{\mathbb{S}} Y=X \wedge Y$ and $\operatorname{Hom}_{\mathbb{S}}(X, Y)=F(X, Y)$.

This section is based on [27, 28]. 
3.A. Koszul spectra. For $\alpha \in \pi_{*} R$, we define the stable Koszul spectrum $K(\alpha)$ by the fibre sequence

$$
K(\alpha) \longrightarrow R \longrightarrow R[1 / \alpha]
$$

where $R[1 / \alpha]=\underset{\rightarrow}{\operatorname{holim}}(R \stackrel{\alpha}{\longrightarrow} R \stackrel{\alpha}{\longrightarrow} \ldots)$. Analogous to the filtration by degree in chain complexes, we obtain a filtration of the $R$-module $K(\alpha)$ by viewing it as

$$
\Sigma^{-1}(R[1 / \alpha] \cup C R) .
$$

Next we define the stable Koszul spectrum for the sequence $\alpha_{1}, \ldots, \alpha_{n}$ by

$$
K\left(\alpha_{1}, \ldots, \alpha_{n}\right)=K\left(\alpha_{1}\right) \otimes_{R} \cdots \otimes_{R} K\left(\alpha_{n}\right),
$$

and give it the tensor product filtration.

The topological analogue of Lemma 2.1 states that if $\beta \in I$ then

$$
K\left(\alpha_{1}, \ldots, \alpha_{n}\right)[1 / \beta] \simeq 0
$$

this follows from Lemma 2.1 and the spectral sequence (3) below. We may now use precisely the same proof as in the algebraic case to conclude that the homotopy type of $K\left(\alpha_{1}, \ldots, \alpha_{n}\right)$ depends only on the radical of the ideal $I=\left(\alpha_{1}, \cdots, \alpha_{n}\right)$. We therefore write $K(I)$ for $K\left(\alpha_{1}, \ldots, \alpha_{n}\right)$.

3.B. Localization and completion. With motivation from Theorems 2.5 and 2.6] we define the homotopical $I$-power torsion (or local cohomology) and homotopical completion (or local homology) modules associated to an $R$-module $M$ by

$$
\Gamma_{I}(M)=K(I) \otimes_{R} M \quad \text { and } \quad \Lambda_{I}(M)=M_{I}^{\wedge}=\operatorname{Hom}_{R}(K(I), M) .
$$

In particular, $\Gamma_{I}(R)=K(I)$.

Because the construction follows the algebra so precisely, it is easy give methods of calculation for the homotopy groups of these $R$-modules. We use the product of the filtrations of the $K\left(\alpha_{i}\right)$ given above and obtain spectral sequences

$$
E_{s, t}^{2}=H_{I}^{-s,-t}\left(R_{*} ; M_{*}\right) \Rightarrow \pi_{s+t}\left(\Gamma_{I} M\right)
$$

with differentials $d^{r}: E_{s, t}^{r} \rightarrow E_{s-r, t+r-1}^{r}$ and

$$
E_{2}^{s, t}=H_{-s,-t}^{I}\left(R^{*} ; M^{*}\right) \Rightarrow \pi_{-(s+t)}\left(M_{I}^{\wedge}\right)
$$

with differentials $d_{r}: E_{r}^{s, t} \rightarrow E_{r}^{s+r, t-r+1}$.

3.C. The Čech spectra. Similarly, we define the Čech spectrum by the cofibre sequence

$$
K(I) \longrightarrow R \longrightarrow \check{C}(I) \text {. }
$$


We define the homotopical localization (or Čech cohomology) and Čech homology modules associated to an $R$-module $M$ by

$$
M\left[I^{-1}\right]=\check{C}(I) \otimes_{R} M \quad \text { and } \quad \Delta^{I}(M)=\operatorname{Hom}_{R}(\check{C}(I), M) .
$$

In particular, $R\left[I^{-1}\right]=\check{C}(I)$. Once again, we have spectral sequences for calculating their homotopy groups from the analogous algebraic constructions.

3.D. Basic properties. We can now give topological analogues of some basic pieces of algebra that we used in Section 2 Recall that the algebraic Koszul complex $K^{\bullet}(I)$ is a direct limit of unstable complexes $K_{s}^{\bullet}(I)$ that are finite complexes of free modules with homology annihilated by a power of $I$. We say that an $R$-module $M$ is a $I$-power torsion module if its $R_{*}$-module $M_{*}$ of homotopy groups is a $I$-power torsion module; equivalently, $M_{*}$ must have support over $I$.

Lemma 3.1. The R-module $K(I)$ is a homotopy direct limit of finite $R$-modules $K_{s}(I)$, each of which has homotopy groups annihilated by some power of $I$. Therefore $K(I)$ is a $I$-power torsion module.

Proof: It is enough to establish the result for a principal ideal and then take tensor products over $R$. Exactly as in the algebraic context, since $K(\alpha)$ is the fibre of $R \longrightarrow R[1 / \alpha]$, we find $K(\alpha) \simeq \operatorname{holim}_{s} K_{s}(\alpha)$, where $K_{s}(\alpha)=\Sigma^{-1} R / \alpha^{s}$ is the fibre of $\alpha^{s}: R \longrightarrow R$.

The following lemma is an analogue of the fact that $\check{C}^{\bullet}(I)$ is a chain complex which is a finite sum of modules $R[1 / \alpha]$ for $\alpha \in I$.

Lemma 3.2. The R-module $\check{C}(I)$ has a finite filtration by $R$-submodules with subquotients that are suspensions of modules of the form $R[1 / \alpha]$ with $\alpha \in I$.

Proof: By construction $K(\alpha)=\Sigma^{-1} R[1 / \alpha] \cup C R$, giving it a filtration of length 1 . The tensor product $K(I)$ therefore has a filtration of length $n$ with the top quotient $R$ and all other subquotients of the form $R[1 / \alpha]$ for $\alpha \in I$. The result follows since $\check{C}(I)$ is the mapping cone of $K(I) \longrightarrow R$.

These lemmas are useful in combination.

COROLlaRY 3.3. If $M$ is a I-power torsion module then $M \otimes_{R} \check{C}(I) \simeq 0$; in particular $K(I) \otimes_{R} \check{C}(I) \simeq 0$.

Proof: Since $M[1 / \alpha] \simeq 0$ for $\alpha \in I$, Lemma 3.2 gives the conclusion for $M$. 


\section{Completion at ideals and Bousfield localization}

Bousfield localizations include both completions at ideals and localizations at multiplicatively closed sets, but one may view these Bousfield localizations as falling into the types typified by completion at $p$ and localization away from $p$. Thinking in terms of $\operatorname{Spec}\left(R_{*}\right)$, this is best viewed as the distinction between localization at a closed set and localization at the complementary open subset. In this section we deal with the closed sets and with the open sets in Section [5] The section is based on $34,35,28$.

4.A. Homotopical completion. As observed in the proof of Lemma 3.1 we have $K(\alpha)=\underset{\rightarrow}{\rightarrow} \Sigma_{s} \Sigma^{-1} R / \alpha^{s}$ and therefore

$$
M_{(\alpha)}^{\wedge}=\operatorname{Hom}_{R}\left(\operatorname{holim}_{\rightarrow} \Sigma^{-1} R / \alpha^{s}, M\right) \simeq \underset{\leftarrow}{\operatorname{holim}} M / \alpha^{s} .
$$

If $I=(\alpha, \beta)$, then

$$
\begin{aligned}
M_{I}^{\wedge} & =\operatorname{Hom}_{R}\left(K(\alpha) \otimes_{R} K(\beta), M\right) \\
& =\operatorname{Hom}_{R}\left(K(\alpha), \operatorname{Hom}_{R}(K(\beta), M)\right) \\
& =\left(M_{(\beta)}^{\wedge}\right)_{(\alpha)}^{\wedge},
\end{aligned}
$$

and so on inductively. This should help justify the notation $M_{I}^{\wedge}=\operatorname{Hom}_{R}(K(I), M)$.

When $R=\mathbb{S}$ is the sphere spectrum and $p \in \mathbb{Z} \cong \pi_{0}(\mathbb{S}), K(p)$ is a Moore spectrum for $\mathbb{Z} / p^{\infty}$ in degree -1 and we recover the usual definition

$$
X_{p}^{\wedge}=F\left(S^{-1} / p^{\infty}, X\right)
$$

of $p$-completions of spectra as a special case, where $F(A, B)=\operatorname{Hom}_{\mathbb{S}}(A, B)$ is the function spectrum. The standard short exact sequence for the calculation of the homotopy groups of $X_{p}^{\wedge}$ in terms of 'Ext completion' and 'Hom completion' follows directly from Corollary 2.8

Since $p$-completion has long been understood to be an example of a Bousfield localization, our next task is to show that completion at $I$ is a Bousfield localization in general.

4.B. Bousfield's terminology. Fix an $R$-module $E$. A spectrum $A$ is $E$ acyclic if $A \otimes_{R} E \simeq 0$; a map $f: X \longrightarrow Y$ is an E-equivalence if its cofibre is $E$-acyclic. An $R$-module $M$ is $E$-local if $E \otimes_{R} T \simeq 0$ implies $\operatorname{Hom}_{R}(T, M) \simeq 0$. A map $Y \longrightarrow L_{E} Y$ is a Bousfield E-localization of $Y$ if it is an $E$-equivalence and $L_{E} Y$ is $E$-local. This means that $Y \longrightarrow L_{E} Y$ is terminal among $E$-equivalences with domain $Y$, and the Bousfield localization is therefore unique if it exists. Similarly, we may replace the single spectrum $E$ by a class $\mathcal{E}$ of objects $E$, and require the conditions hold for all such $E$

The following is a specialization of a change of rings result to the ring map $\mathbb{S} \longrightarrow R$. 
Lemma 4.1. Let $\mathcal{E}$ be a class of $R$-modules. If an $R$-module $N$ is $\mathcal{E}$-local as an $R$-module, then it is $\mathcal{E}$-local as an $\mathbb{S}$-module.

Proof: If $E \wedge T=E \otimes_{\mathbb{S}} T \simeq *$ for all $E$, then $E \otimes_{R}\left(R \otimes_{\mathbb{S}} T\right) \simeq 0$ for all $E$ and therefore $F(T, N)=\operatorname{Hom}_{\mathbb{S}}(T, N) \simeq \operatorname{Hom}_{R}\left(R \otimes_{\mathbb{S}} T, N\right) \simeq 0$.

4.C. Homotopical completion is a Bousfield localization. The class that will concern us most is the class $I$-Tors of finite $I$-power torsion $R$-modules $M$. Thus $M$ must be a finite cell $R$-module, and its $R_{*}$-module $M_{*}$ of homotopy groups must be a $I$-power torsion module.

TheOrem 4.2. For any finitely generated ideal $I$ of $R_{*}$ the map $M \longrightarrow M_{I}^{\wedge}$ is Bousfield localization in the category of $R$-modules in each of the following equivalent senses:

(i) with respect to the $R$-module $\Gamma_{I}(R)=K(I)$.

(ii) with respect to the class $I$-Tors of finite $I$-power torsion $R$-modules.

(iii) with respect to the $R$-module $K_{s}(I)$ for any $s \geq 1$.

Furthermore, the homotopy groups of the completion are related to local homology groups by a spectral sequence

$$
E_{s, t}^{2}=H_{s, t}^{I}\left(M_{*}\right) \Longrightarrow \pi_{s+t}\left(M_{I}^{\wedge}\right) .
$$

If $R_{*}$ is Noetherian, the $E^{2}$ term consists of the left derived functors of I-adic completion: $H_{s}^{I}\left(M_{*}\right)=L_{s}^{I}\left(M_{*}\right)$.

Proof: We begin with (i). Since

$$
\operatorname{Hom}_{R}\left(T, M_{I}^{\wedge}\right) \simeq \operatorname{Hom}_{R}\left(T \otimes_{R} K(I), M\right),
$$

it is immediate that $M_{I}^{\wedge}$ is $K(I)$-local. We must prove that the map $M \longrightarrow M_{I}^{\wedge}$ is a $K(I)$-equivalence. The fibre of this map is $\operatorname{Hom}_{R}(\check{C}(I), M)$, so we must show that

$$
\operatorname{Hom}_{R}(\check{C}(I), M) \otimes_{R} K(I) \simeq 0 .
$$

By Lemma $3.1 K(I)$ is a homotopy direct limit of terms $K_{s}(I)$. Each $K_{s}(I)$ is in $I$-Tors, and we see by their definition in terms of cofibre sequences and smash products that their duals $K_{s}(I)^{\#}$ are also in $I$-Tors, where $M^{\#}=\operatorname{Hom}_{R}(M, R)$. Since $K_{s}(I)$ is a finite cell $R$-module,

$$
\operatorname{Hom}_{R}(\check{C}(I), M) \otimes_{R} K_{s}(I)=\operatorname{Hom}_{R}\left(\check{C}(I) \otimes_{R} K_{s}(I)^{\#}, M\right),
$$

and $\check{C}(I) \otimes_{R} K_{s}(I)^{\#} \simeq 0$ by Corollary 3.3 Parts (ii) and (iii) are similar but simpler. For (iii), observe that we have a cofibre sequence $R / \alpha^{s} \longrightarrow R / \alpha^{2 s} \longrightarrow R / \alpha^{s}$, so that all of the $K_{j s}(I)$ may be constructed from $K_{s}(I)$ using a finite number of cofibre sequences. 


\section{Localization away from ideals and Bousfield localization}

In this section we turn to localization away from the closed set defined by an ideal $I$. First, observe that, when $I=(\alpha), M\left[I^{-1}\right]$ is just $R\left[\alpha^{-1}\right] \otimes_{R} M=M\left[\alpha^{-1}\right]$. However, the higher Čech cohomology groups give the construction for general finitely generated ideals a quite different algebraic flavour, and $M\left[I^{-1}\right]$ is rarely a localization of $M$ at a multiplicatively closed subset of $R_{*}$. The section is based on 28 .

5.A. The Čech complex as a Bousfield localization. To characterize this construction as a Bousfield localization, we consider the class $I$-Inv of $R$-modules $M$ for which there is an element $\alpha \in I$ such that $\alpha: M \longrightarrow M$ is an equivalence.

TheOREM 5.1. For any finitely generated ideal $I=\left(\alpha_{1}, \ldots, \alpha_{n}\right)$ of $R_{*}$, the map $M \longrightarrow M\left[I^{-1}\right]$ is Bousfield localization in the category of $R$-modules in each of the following equivalent senses:

(i) with respect to the $R$-module $R\left[I^{-1}\right]=\check{C}(I)$.

(ii) with respect to the class $I$-Inv.

(iii) with respect to the set $\left\{R\left[1 / \alpha_{1}\right], \ldots, R\left[1 / \alpha_{n}\right]\right\}$.

Furthermore, the homotopy groups of the localization are related to Čech cohomology groups by a spectral sequence

$$
E_{s, t}^{2}=\check{C} H_{I}^{-s,-t}\left(M_{*}\right) \Longrightarrow \pi_{s+t}\left(M\left[I^{-1}\right]\right) .
$$

If $R_{*}$ is Noetherian, the $E^{2}$ term can be viewed as the cohomology of $\operatorname{Spec}\left(R_{*}\right) \backslash V(I)$ with coefficients in the sheaf associated to $M_{*}$.

Proof: We prove Part (i); Parts (ii) and (iii) are proved similarly. To see that $M\left[I^{-1}\right]$ is local, suppose that $T \otimes_{R} \check{C}(I) \simeq 0$. We must show that $\operatorname{Hom}_{R}\left(T, M\left[I^{-1}\right]\right) \simeq$ 0 . By the cofibre sequence defining $\check{C}(I)$ and the supposition, it suffices to show that $\operatorname{Hom}_{R}\left(K(I) \otimes_{R} T, M\left[I^{-1}\right]\right) \simeq 0$. By Lemma 3.1

$$
\operatorname{Hom}_{R}\left(K(I) \otimes_{R} T, M\left[I^{-1}\right]\right) \simeq \underset{\leftarrow}{\operatorname{holim}} \operatorname{Hom}_{R}\left(K_{s}(I) \otimes_{R} T, \check{C}(I) \otimes_{R} M\right) .
$$

Observing that

$$
\operatorname{Hom}_{R}\left(K_{s}(I) \otimes_{R} T, \check{C}(I) \otimes_{R} M\right) \simeq \operatorname{Hom}_{R}\left(T, K_{s}(I)^{\#} \otimes_{R} \check{C}(I) \otimes_{R} M\right),
$$

we see that the conclusion follows from Corollary 3.3 The map $M \longrightarrow M\left[I^{-1}\right]$ is a $\check{C}(I)$-equivalence since its fibre is $\Gamma_{I}(M)=K(I) \otimes_{R} M$ and $K(I) \otimes_{R} \check{C}(I) \simeq 0$ by Corollary 3.3 
REMARK 5.2. Translating the usual terminology, we say that a localization $L$ on $R$-modules is smashing if $L(N)=N \otimes_{R} L(R)$ for all $R$-modules $N$. It is clear that localization away from $I$ is smashing and that completion at $I$ will usually not be smashing. For any smashing localization one may form an associated complementary completion, giving a formal situation like the present one $\mathbf{3 0}$.

REMARK 5.3. One may also characterize the map $\Gamma_{I}(M) \longrightarrow M$ by a universal property analogous to that of the cellular approximation in spaces.

On the one hand, $\Gamma_{I}(M)$ is constructed from $K_{1}(I)$ by 3.1 and on the other hand, the map induces an equivalence of $\operatorname{Hom}_{R}\left(K_{1}(I), \cdot\right)$ since, by Lemma 3.2 $\operatorname{Hom}_{R}\left(K_{1}(I), \check{C}(I)\right) \simeq 0$. We will return to these ideas in Part 2 .

\section{Chromatic filtrations for $M U$-modules.}

We have described ways of picking out parts of stable homotopy theory concentrated on open and closed subschemes of a coefficient ring. This philosophy was used before in stable homotopy theory in a deeper way, where the analogy needed much more work to set up. We show in this section that the two approaches agree where they overlap.

The starting point of the chromatic apprach to homotopy theory is that one may attempt to make calculations in stable homotopy theory using a chosen cohomology theory. One of the most effective is complex cobordism $M U^{*}(X)$. Not only is it a very practical means of calculation in many cases, but it also leads to remarkable structural insight [4, 49, 51, 45. Indeed, the Adams-Novikov spectral sequence gives a means of calculation in the category of $\mathbb{S}$-modules based on the category of $M U_{*}$-modules. However the connection goes further. The coefficient ring $M U_{*}$ is isomorphic Lazard's universal ring for 1-dimensional commutative formal group laws, and Quillen proved that there is a canonical isomorphism for geometric reasons. Thus the algebra of $M U_{*}$-modules is that of 1-dimensional commutative formal groups, and this gives rise to a filtration by height. This algebraic filtration in turn gives rise to the chromatic filtration of stable homotopy.

Since cobordism is represented by a commutative $\mathbb{S}$-algebra $M U$, there is a wellbehaved category of $M U$-modules, and we may lift the Adams-Novikov spectral sequence to the level of $M U$-modules. The formalities we have described in Section 3 above give a filtration of the category of $M U$-modules, and it is the purpose of this section to explain that this agrees with the restriction of the chromatic filtration, and to provide a dictionary for relating the languages.

6.A. Cobordism notation. Recall that $M U_{*}=\mathbb{Z}\left[x_{i} \mid i \geq 1\right]$, where $\operatorname{deg}\left(x_{i}\right)=$ $2 i$, and that $M U_{*}$ contains elements $v_{i}$ of degree $2\left(p^{i}-1\right)$ that map to the Hazewinkel generators of $B P_{*}=\mathbb{Z}_{(p)}\left[v_{i} \mid i \geq 1\right]$. We let $I_{n}$ denote the ideal $\left(v_{0}, v_{1}, \ldots, v_{n-1}\right)$ in 
$\pi_{*}(M U)$, where $v_{0}=p$; we work with $M U$ rather than $B P$ because of its canonical $\mathbb{S}$-algebra structure. The Brown-Peterson espectrum $B P$ is a ring in the homotopy category of $M U$-modules whose unit $M U \longrightarrow B P$ factors through the canonical retraction $M U_{(p)} \longrightarrow B P$. There are also $M U$-module spectra $E(n)$ and $K(n)$ such that $E(0)_{*}=K(0)_{*}=\mathbb{Q}$, and for $n \geq 1$

$$
K(n)_{*}=\mathbb{F}_{p}\left[v_{n}, v_{n}^{-1}\right] \text { and } E(n)_{*}=\mathbb{Z}_{(p)}\left[v_{1}, \ldots, v_{n}, v_{n}^{-1}\right] .
$$

Furthermore $E(n)$ is a ring in the homotopy category of $M U$-modules.

6.B. Chromatic filtration and the Cousin complex. The chromatic filtration corresponds to the Bousfield localizations $L_{n}$ with respect to $K(0) \vee K(1) \vee$ $\ldots \vee K(n)$, or equivalently with respect to $E(n)$. For any spectrum $X$, Ravenel defines the $n$th acyclization $C_{n} X$ and the $n$th monochromatic part $M_{n} X$ by the cofibrations

$$
C_{n} X \longrightarrow X \longrightarrow L_{n} X
$$

and

$$
\Sigma^{-n} M_{n} X \longrightarrow L_{n} X \longrightarrow L_{n-1} X .
$$

To start inductions, $L_{-1} X=*$.

The connection between the present constructions and Ravenel's are provided by the following dictionary.

Lemma 6.1. For an $M U$-module $A$, and for any $n \geq-1$, we have

$$
\begin{aligned}
L_{n} A & \simeq A\left[I_{n+1}^{-1}\right], \\
C_{n} A & \simeq \Gamma_{I_{n+1}} A
\end{aligned}
$$

and

$$
M_{n} A \simeq \Sigma^{n-1} \Gamma_{I_{n}} A\left[1 / v_{n}\right] .
$$

Proof: It suffices to establish the first identification. Indeed, $C_{n} A$ and $M_{n} A$ are then defined by cofibre sequences from the $C_{i}$, and these are precise counterparts of

$$
\Gamma_{I_{n+1}} A \longrightarrow A \longrightarrow A\left[I_{n+1}^{-1}\right]
$$

and

$$
\Sigma^{-1} \Gamma_{I_{n}} A\left[1 / v_{n}\right] \longrightarrow \Gamma_{I_{n+1}} A \longrightarrow \Gamma_{I_{n}} A
$$

from Subsection 2.G

The idea of the comparison is clear, but the obstacle is that in considering $M U_{*}(X)$ for an $M U$-module $X$ we must relate the $M U_{*}$-action through $M U$ to the $M U_{*}$-action through $X$. We need to engineer a situation where we can apply the well-known formulae this purpose. By [51, 7.3.2], localization at $E(n)$ is the same as localization at the wedge of $M U\left[1 / v_{i}\right]$ for $0 \leq i \leq n$. By 4.1 we conclude that $M\left[I_{n+1}^{-1}\right]$ is $E(n)$-local. To see that $M \longrightarrow M\left[I_{n+1}^{-1}\right]$ is an $M U\left[1 / v_{i}\right]$-equivalence 
for $0 \leq i \leq n$, note that its fibre is $\Gamma_{I_{n+1}} M$ and $\Gamma_{I_{n+1}} M[1 / w] \simeq 0$ for any $w \in I_{n+1}$. Consider $\pi_{*}(M U \wedge M U)$ as a left $M U_{*}$-module, and recall from [51, B.5.15] that the right unit $\eta_{R}: M U_{*} \longrightarrow \pi_{*}(M U \wedge M U)$ satisfies

$$
\eta_{R}\left(v_{i}\right) \equiv v_{i} \bmod I_{i} \cdot \pi_{*}(M U \wedge M U) \text {, hence } \eta_{R}\left(v_{i}\right) \in I_{i+1} \cdot \pi_{*}(M U \wedge M U) .
$$

We have

$$
\Gamma_{I_{n+1}}(M) \wedge M U \simeq \Gamma_{I_{n+1}}(M) \otimes_{M U}(M U \wedge M U)
$$

and can deduce inductively that $\Gamma_{I_{n+1}} M \wedge M U[1 / w] \simeq 0$ for any $w \in I_{n+1}$ since $\Gamma_{I_{n+1}}(M)[1 / w] \simeq 0$ for any such $w$.

6.C. Chromatic completions of $M U$-modules and $\mathbb{S}$-modules. We first recall the definition of the chromatic completion. For a sequence $\mathbf{i}=\left(i_{0}, i_{1}, \ldots, i_{n-1}\right)$, we may attempt to construct generalized Toda-Smith spectra

$$
M_{\mathbf{i}}=M\left(p^{i_{0}}, v_{1}^{i_{1}}, \ldots, v_{n-1}^{i_{n-1}}\right)
$$

inductively, starting with $\mathbb{S}$, continuing with the cofibre sequence

$$
M\left(p^{i_{0}}\right) \longrightarrow \mathbb{S} \stackrel{p^{i_{0}}}{\longrightarrow} \mathbb{S}
$$

and, given $L=M_{\left(i_{0}, i_{1}, \ldots, i_{n-2}\right)}$, concluding with the cofibre sequence

$$
M_{\mathbf{i}} \longrightarrow L \stackrel{v_{n-1}^{i_{n}-1}}{\longrightarrow} L \text {. }
$$

Here $M_{\mathbf{i}}$ is a finite complex of type $n$ and hence admits a $v_{n}$-self map by the Nilpotence Theorem [16, 44, and $v_{n}^{i_{n}}$ is shorthand for such a map. These spectra do not exist for all sequences $\mathbf{i}$, but they do exist for a cofinal set of sequences, and Devinatz has shown [15] that there is a cofinal collection all of which are ring spectra. These spectra are not determined by the sequence, but it follows from the Nilpotence Theorem that they are asymptotically unique in the sense that $\underset{\rightarrow}{\operatorname{holim}} M_{\mathbf{i}}$ is independent of all choices. Hence we may define a completion for all $p$-local spectra $X$ by

$$
X_{I_{n}}^{\wedge}=F\left(\underset{\rightarrow}{\operatorname{holim}} M_{\mathbf{i}}, X\right)
$$

We denote the spectrum $\operatorname{holim}_{\rightarrow} M_{\mathbf{i}}$ by $\Gamma_{I_{n}}(\mathbb{S})$, although it is not simply a local cohomology spectrum.

Proposition 6.2. Localize all spectra at $p$. Then there is an equivalence of MU-modules

$$
M U \wedge \Gamma_{I_{n}}(\mathbb{S}) \simeq \Gamma_{I_{n}}(M U) .
$$

Therefore, for any $M U$-module $M$, there is an equivalence of $M U$-modules between the two completions $M_{I_{n}}^{\wedge}$. 
Proof: The second statement follows from the first since

$$
\operatorname{Hom}_{M U}\left(M U \wedge \Gamma_{I_{n}}(\mathbb{S}), M\right) \simeq F\left(\Gamma_{I_{n}}(\mathbb{S}), M\right)
$$

as $M U$-modules. It suffices to construct compatible equivalences

$$
M U \wedge M_{\mathbf{i}} \simeq M U / p^{i_{0}} \otimes_{M U} M U / v_{1}^{i_{1}} \otimes_{M U} \cdots \otimes_{M U} M U / v_{n-1}^{i_{n-1}} .
$$

The right side is equivalent to $M U / I_{\mathbf{i}}$, where $I_{\mathbf{i}}=\left(p^{i_{0}}, v_{1}^{i_{1}}, \ldots, v_{n-1}^{i_{n-1}}\right) \subset I_{n}$. A $v_{n}$-self map $v: X \longrightarrow X$ on a type $n$ finite complex $X$ can be characterized as a map such that, for some $i, B P_{*}\left(v^{i}\right): B P_{*}(X) \longrightarrow B P_{*}(X)$ is multiplication by $v_{n}^{j}$ for some $j$. Since $M U_{*}(X)=M U_{*} \otimes_{B P_{*}} M U_{*}(X)$, we can use $M U$ instead of $B P$. Using $M U$, we conclude that the two maps of spectra id $\wedge v^{i}$ and $v_{n}^{j} \wedge$ id from $M U \wedge X$ to itself induce the same map on homotopy groups. The cofibre of the first is $M U \wedge C v^{i}$ and the cofibre of the second is $M U /\left(v_{n}^{j}\right) \wedge X$. If $X$ is a generalized Moore spectrum, results of [44] show that some powers of these two maps are homotopic, hence the cofibres of these powers are equivalent. The conclusion follows by induction.

\section{Completion theorems and their duals.}

Finally, we return to the motivating context with a section based on $[\mathbf{3 4}, \mathbf{2 7}$. There is more background on equivariant stable homotopy theory in 36. So as to illustrate the result in the simplest context we choose a finite group $G$, and an equivariant cohomology theory $R_{G}^{*}(\cdot)$ which is Noetherian in the sense that $R_{G}^{*}$ is Noetherian and for any finite $G$-CW-complex $X$, the $R_{G}^{*}$-module $R_{G}^{*}(X)$ is finitely generated. We also suppose that $R_{G}^{*}(X) \cong R^{*}(X / G)$ if $X$ is $G$-free (in fact we make the corresponding assumption about the representing $G$-spectrum, which is to say that it is split [39]). It smooths the exposition to suppose throughout that $G$-spaces are equipped with a $G$-fixed basepoint. This is no loss of generality since an unbased $G$-space $Y$ may be converted to a based $G$-space $Y_{+}$by adding a disjoint basepoint. The unreduced cohomology of $Y$ is the reduced cohomology of $Y_{+}$.

The completion theorem concerns the map

$$
R_{G}^{*}=R_{G}^{*}\left(S^{0}\right) \longrightarrow R_{G}^{*}\left(E G_{+}\right)=R^{*}\left(B G_{+}\right)
$$

induced by projection $E G \longrightarrow p t$, or more generally

$$
R_{G}^{*}(X) \longrightarrow R_{G}^{*}\left(E G_{+} \wedge X\right)=R^{*}\left(E G_{+} \wedge_{G} X\right)
$$

for a finite based $G$-CW-complex $X$. The classical completion theorem is said to hold if these maps are completion at the augmentation ideal

$$
I=\operatorname{ker}\left(R_{G}^{*} \longrightarrow R^{*}\right) .
$$


By the Noetherian condition, $I$ is finitely generated, so if $R_{G}^{*}(\cdot)$ is represented by a strictly commutative ring spectrum, we may form the $I$-adic completion of $R$. The classical completion theorem for the case $X=p t_{+}=S^{0}$ then suggests the derived completion theorem:

$$
F\left(E G_{+}, R\right) \simeq R_{I}^{\wedge} .
$$

More precisely, the statement is that the natural map $R \longrightarrow F\left(E G_{+}, R\right)$ has the universal property of $I$-adic completion.

If the derived completion theorem holds, the classical completion theorem holds by taking homotopy groups, since the higher derived functors vanish by the ArtinRees Lemma. Furthermore, applying $F(X, \cdot)$ we obtain

$$
F\left(X \wedge E G_{+}, R\right) \simeq F(X, R)_{I}^{\wedge} .
$$

The classical completion theorem for a general finite $X$ follows by taking homotopy groups. Furthermore, we can see how to formulate the appropriate statement for an infinite $X$ using local homology 34

Now notice that $R_{I}^{\wedge}=\operatorname{Hom}_{R}\left(\Gamma_{I} R, R\right)$ and $F\left(E G_{+}, R\right) \simeq \operatorname{Hom}_{R}\left(R \wedge E G_{+}, R\right)$. Thus the derived completion theorem is the statement that $\Gamma_{I} R$ and $R \wedge E G_{+}$ become equivalent after applying $\operatorname{Hom}_{R}(\cdot, R)$. Indeed, there are obvious comparison maps

$$
R \wedge E G_{+} \stackrel{\simeq}{\longleftarrow} \Gamma_{I}\left(R \wedge E G_{+}\right) \longrightarrow \Gamma_{I} R,
$$

and it is elementary that the left hand map is an equivalence: the map $\Gamma_{I}(X \wedge$ $F) \longrightarrow X \wedge F$ is an equivalence when $F=G_{+}$since $\operatorname{res}_{1}^{G}(I)=(0)$, and hence for $F=E G_{+}$, since it is constructed from $G$-free cells.

The simplest way that the derived completion could be true would be if the derived local cohomology theorem holds:

$$
R \wedge E G_{+} \simeq \Gamma_{I} R,
$$

or more precisely that $R \wedge E G_{+} \longrightarrow R$ has the universal property of $\Gamma_{I} R \longrightarrow R$.

The remarkable fact is that for complex oriented cohomology theories this strengthening of the completion theorem holds. Indeed, the proof is simple enough that we can outline it here. However there are examples (such as stable cohomotopy) for which the derived completion theorem holds (by Carlsson's proof [14 of the Segal Conjecture) but for which the local cohomology theorem is false.

Theorem 7.1. For a complex oriented, Noetherian theory, the derived local cohomology theorem holds in the sense that

$$
R \wedge E G_{+} \simeq \Gamma_{I} R .
$$

Hence in particular there is a spectral sequence

$$
H_{I}^{*}\left(R_{G}^{*}\right) \Rightarrow R_{*}^{G}\left(E G_{+}\right) \cong R_{*}\left(B G_{+}\right) .
$$


Proof: We need only show that the map $\Gamma_{I}\left(R \wedge E G_{+}\right) \longrightarrow \Gamma_{I}(R)$ is an equivalence, or equivalently that $\Gamma_{I}(R \wedge \tilde{E} G) \simeq 0$, where $\tilde{E} G$ is the mapping cone of $E G_{+} \longrightarrow$ $S^{0}$. Since the theory is complex oriented, we may define Euler clases of complex representations.

If $G$ is of prime order we may take $\tilde{E} G=S^{\infty V}$ where $V$ is the complex reduced regular representation and then use the fact that the Euler class $\chi(V)$ is in $I$, combined with Lemma 2.1

The same argument shows that $\Gamma_{I}\left(R \wedge S^{\infty V}\right) \simeq 0$ for any complex representtion $V$. We use an induction on the group order, noting that if $V$ is the reduced regular representation, there is a map $\tilde{E} G \longrightarrow S^{\infty V}$ whose mapping cone $C$ is built from cells $G / H_{+}$with $H$ a proper subgroup. The Noetherian hypothesis is enough to ensure the radical of $\operatorname{res}_{H}^{G}(I(G))$ is $I(H)$, so it follows by induction that $\Gamma_{I}\left(R \wedge \tilde{E} G \wedge G / H_{+}\right) \simeq 0$, and hence that $\Gamma_{I}(R \wedge \tilde{E} G \wedge C) \simeq 0$ as required.

\section{Part 2. Morita equivalences and Gorenstein rings.}

In Part 1 we considered certain localizations and completions associated to an ideal $I$ of the coefficient ring $R_{*}$ of a ring spectrum $R$, and gave characterizations up to homotopy, but the methods were based on using particular elements of $R_{*}$. In Part 2, we change focus from the pair $(I, R)$ to the pair $\left(R, R_{*} / I\right)$, and then replace the graded ring $R_{*} / I$ by a ring spectrum $k$. This is a strict refinement when the coefficient ring of $k$ is the quotient considered before, in the sense that $k_{*}=R_{*} / I$. Conceptually, the present situation is considerably more general, but for a particular ring spectrum $R$ and a particular ideal $I$ of its coefficients it is sometimes impossible to construct an $R$-module $k$ (let alone an $R$-algebra) with $k_{*}=R_{*} / I$.

To summarize: in Part 2 we consider a map $R \longrightarrow k$ of ring spectra, and the notation is chosen to suggest the analogy with a commutative Noetherian local ring $R$ with residue field $k$. In effect we are extending the idea of trying to do commutative algebra entirely in the derived category. Given the map $R \longrightarrow k$, it turns out that completion and localization arise from a Morita adjunction, so that it is essential that we consider non-commutative rings, even if we only want conclusions in commutative algebra. Part 2 is based on the papers [18 and [19].

\section{The context, and some examples.}

The basic ingredients are as follows. 
Context 8.1. The main input is a map $R \longrightarrow k$ of ring spectra with notation suggested by the case when $R$ is commutative local ring with residue field $k$. We also write $\mathcal{E}=\operatorname{Hom}_{R}(k, k)$ for the (derived) endomorphism ring spectrum.

We work in the derived category $D(R$-mod) of left $R$-modules.

8.A. New modules from old. Three construction principles will be important to us. There is some duplication in terminology, but the flexibility is convenient.

If $M$ is an $R$-module we say that $X$ is built from $M$ if $X$ can be formed from $M$ by completing triangles, taking coproducts and retracts (i.e., $X$ is in the localizing subcategory generated by $M$ ). We refer to objects built from $M$ as $M$-cellular, and write $\operatorname{Cell}(R, M)$ for the resulting full subcategory of $D(R$-mod). An $M$-cellular approximation of $X$ is a map $\operatorname{Cell}_{M}(X) \longrightarrow X$ where $\operatorname{Cell}_{M}(X)$ is $M$-cellular and the map is an $\operatorname{Hom}_{R}(M, \cdot)$-equivalence.

We say that it is finitely built from $M$ if only finitely many steps and finite coproducts are necessary (i.e., $X$ is in the thick subcategory generated by $M$ ).

Finally, we say that $X$ is cobuilt from $M$ if $X$ can be formed from $M$ by completing triangles, taking products and retracts (i.e., $X$ is in the colocalizing subcategory generated by $M$ ).

8.B. Finiteness conditions. We say $M$ is small if it is finitely built from $R$ and we say $R$ is regular if $k$ is small as an $R$-module. The theory is easiest if $R$ is regular, but this assumption is very strong. We will develop a useful theory under a much weaker condition.

Definition 8.2. 19] We say that $k$ is proxy-small if there is an object $K$ with the following properties

- $K$ is small

- $K$ is finitely built from $k$ and

- $k$ is built from $K$.

REMARK 8.3. Note that the second and third condition imply that the $R$ module $K$ generates the same category as $k$ using triangles and coproducts: $\operatorname{Cell}(R, K)=$ $\operatorname{Cell}(R, k)$.

It is one of the messages of $[\mathbf{1 9}$ that the weak condition of proxy-smallness allows one to develop a very useful theory.

8.C. The principal examples. We will illustrate the theory throughout with three rather extensive families of examples, giving them in this order each time. 
ExAmPles 8.4. (i) (Local algebra) Take $R$ to be a commutative Noetherian local ring in degree 0 , with maximal ideal $I$ and residue field $k$.

By the Auslander-Buchsbaum-Serre theorem, $k$ is small if and only if $R$ is a regular local ring, confirming that the smallness of $k$ is a very strong condition. On the other hand, $k$ is always proxy-small: we may take $K=K_{1}(\boldsymbol{\alpha})$ to be the Koszul complex for a generating sequence $\boldsymbol{\alpha}$ for $I$.

It is shown in 18 that $\operatorname{Cell}(R, k)$ consists of objects whose homology is $I$-power torsion.

The endomorphism $\operatorname{ring} \mathcal{E}$ is modelled by the endomorphism ring of a projective $R$-resolution of the $R$-module $k$. Its homology is the Ext algebra

$$
H_{*}(\mathcal{E})=\operatorname{Ext}_{R}^{*}(k, k) .
$$

(ii) (Cochains on a space) For a field $k$, consider the cochain complex for a space $X$

$$
R=C^{*}(X ; k) .
$$

We want a commutative model, so it is not possible to use a conventional differential graded algebra unless $k$ is of characteristic 0 . Instead, we identify the ring $k$ with the associated Eilenberg-MacLane spectrum $H k$, chosen to be a strictly commutative ring spectrum. We will therefore interpret $C^{*}(X ; k)=F(X, H k)$ as the function spectrum of maps from $X$ to the commutative ring spectrum $H k$, and as such it is itself a commutative $k$-algebra spectrum. This is reasonable since $C^{*}(X ; k)$ is a model for ordinary cohomology in the sense that $\pi_{*}\left(C^{*}(X ; k)\right)=H^{*}(X ; k)$.

If the Eilenberg-Moore spectral sequence converges then

$$
\mathcal{E} \simeq C_{*}(\Omega X ; k)
$$

For instance this holds if $X$ is simply connected, or if $k$ is characteristic $p$ and $X$ is connected with fundamental group a finite $p$-group [17.

Under this assumption one may show that $R$ is regular if and only if $H_{*}(\Omega X ; k)$ is finite dimensional. In one direction, we use the Eilenberg-Moore construction: if $k$ is finitely built from $R$ then we apply $\operatorname{Hom}_{R}(\cdot, k)$ to deduce $\operatorname{Hom}_{R}(k, k) \simeq$ $C_{*}(\Omega X ; k)$ is finitely built from $\operatorname{Hom}_{R}(R, k)=k$. The other direction uses the Rothenberg-Steenrod construction. The ring $R$ is proxy-regular much more generally. In particular $R$ is proxy-regular if $X=B G$ for a compact Lie group $G$ or if $X$ is a connected finite complex, whatever the fundamental groups.

(iii) (Equivariant cohomology) To make contact with the equivariant motivation in Part 1 we suppose given a commutative ring $G$-spectrum, $R$ representing $R_{G}^{*}(X)=[X, R]_{G}^{*}$, and consider $k=F\left(G_{+}, R\right)$ representing the non-equivariant theory $R^{*}(X)=[X, R]^{*}=\left[X, F\left(G_{+}, R\right)\right]_{G}^{*}$. The collapse map $G_{+} \longrightarrow S^{0}$ induces 
a ring map $R \longrightarrow k$ and

$$
\mathcal{E}=\operatorname{Hom}_{R}(k, k) \simeq \operatorname{Hom}_{R}\left(F\left(G_{+}, R\right), F\left(G_{+}, R\right)\right) \simeq F\left(G_{+}, G_{+}\right) \wedge R .
$$

The category $\operatorname{Cell}(R, k)$ consists of the $G$-free $R$-modules.

\section{Morita equivalences.}

Morita theory studies objects $X$ of a category $\mathbb{C}$ by considering their counterparts $\operatorname{Hom}(k, X)$ as modules over the endomorphism ring $\operatorname{End}(k)$ of an object $k$. In favourable circumstances this may provide an equivalence between $\mathbb{C}$ and a category of $\operatorname{End}(k)$-modules. Classically, $\mathbb{C}$ is an abelian category with infinite sums and $k$ is a small projective generator, and we find $\mathbb{C}$ is equivalent to the category of $\operatorname{End}(k)$-modules [7, II Thm 1.3]. However there are numerous variations and extensions. The differences in our context that are most significant are that we work in a derived category (or its model) rather than in an abelian category, and that $k$ is not necessarily either small or a generator. The fact that the objects of the categories are spectra is unimportant except for the formal context it provides. See 53 for an account from the present point of view.

Two separate Morita equivalences play a role. It is striking that even where we want conclusions in a commutative context, the Morita equivalences involve us in thinking about non-commutative rings. This may seem less surprising if we think of it as a refinement of the well established use of non-commutative rings of operations in stable homotopy theory. This section is based on [18, with augmentations from 19 .

9.A. First variant. Continuing to let $\mathcal{E}=\operatorname{Hom}_{R}(k, k)$ denote the (derived) endomorphism ring, we consider the relationship between the derived categories of left $R$-modules and of right $\mathcal{E}$-modules. We have the adjoint pair

$$
T: D(\bmod -\mathcal{E}) \rightleftarrows D(R-\bmod ): E
$$

defined by

$$
T(X):=X \otimes_{\mathcal{E}} k \text { and } E(M):=\operatorname{Hom}_{R}(k, M) .
$$

REMARK 9.1. If $k$ is small, it is easy to see that this adjunction gives equivalence

$$
\operatorname{Cell}(R, k) \simeq D(\bmod -\mathcal{E})
$$

between the derived category of $R$-modules built from $k$ and the derived category of $\mathcal{E}$-modules. Indeed, to see the unit $X \longrightarrow E T X=\operatorname{Hom}_{R}\left(k, X \otimes_{\mathcal{E}} k\right)$ is an equivalence, we note it is obviously an equivalence for $X=\mathcal{E}$ and hence for any $X$ built from $\mathcal{E}$, by smallness of $k$. The argument for the counit is similar. 
REMARK 9.2. The unit of the adjunction is not an equivalence in general. For example if $R=\Lambda(\tau)$ is exterior on a generator of degree 1 then $\mathcal{E} \simeq k[x]$ is polynomial on a generator of degree -2 . As an $R$-module, $k$ is of infinite homological dimension and hence it is not small. In this case all $R$-modules are $k$-cellular, so that $\operatorname{Cell}(R, k)=R$-mod. Furthermore, the only subcategories of $R$-modules closed under coproducts and triangles are the trivial category and the whole category. On the other hand the category of torsion $\mathcal{E}$-modules is a proper non-trivial subcategory closed under coproducts and triangles.

Exchanging roles of the rings, so that $R=k[x]$ and $\mathcal{E} \simeq \Lambda(\tau)$, we see $k$ is small as a $k[x]$-module and $\operatorname{Cell}(k[x], k)$ consists of torsion modules. Thus we deduce

$$
\text { tors } k[x]-\bmod \simeq \bmod -\Lambda(\tau) .
$$

In fact the counit

$$
T E M=\operatorname{Hom}_{R}(k, M) \otimes_{\mathcal{E}} k \longrightarrow M
$$

of the adjunction is of interest much more generally. Notice that any $\mathcal{E}$-module (such as $\left.\operatorname{Hom}_{R}(k, M)\right)$ is built from $\mathcal{E}$, so the domain is $k$-cellular. We say $M$ is effectively constructible from $k$ if the counit is an equivalence, because TEM gives a concrete and functorial model for the cellular approximation to $M$. Under the much weaker assumption of proxy smallness we obtain a very useful conclusion linking Morita theory to commutative algebra.

Lemma 9.3. Provided $k$ is proxy-small, the counit

$$
T E M=\operatorname{Hom}_{R}(k, M) \otimes_{\mathcal{E}} k \longrightarrow M
$$

is $k$-cellular approximation, and hence in particular any $k$-cellular object is effectively constructible from $k$.

Proof: We observed above that the domain is $k$-cellular. To see the counit is a $\operatorname{Hom}_{R}(k, \cdot)$-equivalence, consider the evaluation map

$$
\gamma: \operatorname{Hom}_{R}(k, X) \otimes_{\mathcal{E}} \operatorname{Hom}_{R}(Y, k) \longrightarrow \operatorname{Hom}_{R}(Y, X) .
$$

This is an equivalence if $Y=k$, and hence by proxy-smallness it is an equivalence if $Y=K$. This shows that the top horizontal in the diagram

$$
\begin{array}{rlc}
\operatorname{Hom}_{R}(k, X) \otimes_{\mathcal{E}} \operatorname{Hom}_{R}(K, k) & \stackrel{\simeq}{ } & \operatorname{Hom}_{R}(K, X) \\
& & \downarrow= \\
\operatorname{Hom}_{R}\left(K, \operatorname{Hom}_{R}(k, X) \otimes_{\mathcal{E}} k\right) & \longrightarrow & \operatorname{Hom}_{R}(K, X)
\end{array}
$$

is an equivalence. The left hand-vertical is an equivalence since $K$ is small. Thus the lower horizontal is an equivalence, which is to say that the counit

$$
T E X=\operatorname{Hom}_{R}(k, X) \otimes_{\mathcal{E}} k \longrightarrow X
$$

is a $K$-equivalence. By proxy-smallness, this counit map is a $k$-equivalence. 
ExAMPLes 9.4. (i) If $R$ is a commutative local ring, we saw in 5.3 that the $k$-cellular approximation of a module $M$ is $\Gamma_{I} M=K(I) \otimes_{R} M$, so we have

$$
T E M \simeq K(I) \otimes_{R} M .
$$

(ii) If $R=C^{*}(X ; k)$ it is not easy to say what the $k$-cellular approximation is in general, but any bounded below module $M$ is cellular.

(iii) In the equivariant case with $k=F\left(G_{+}, R\right)$ we see that the $k$-cellular approximation of an $R$-module $M$ is $M \wedge E G_{+}$. Even without knowing that $k$ is proxy-small, it is not hard to see that every $G$-free module is effectively constructible.

9.B. Second variant. There is a second adjunction between the derived categories of left $R$-modules and of right $\mathcal{E}$-modules. In the first variant, $k$ played a central role as a left $R$-module and a left $\mathcal{E}$-module. In this second variant

$$
k^{\#}:=\operatorname{Hom}_{R}(k, R)
$$

plays a corresponding role: it is a right $R$-module and a right $\mathcal{E}$-module. We have the adjoint pair

$$
E^{\prime}: D(R-\bmod ) \rightleftarrows D(\bmod -\mathcal{E}): C
$$

defined by

$$
E^{\prime}(M):=k^{\#} \otimes_{R} M \text { and } C(X):=\operatorname{Hom}_{\mathcal{E}}\left(k^{\#}, X\right) .
$$

REMARK 9.5. If $k$ is small then

$$
E^{\prime}(M)=\operatorname{Hom}_{R}(k, R) \otimes_{R} M \simeq \operatorname{Hom}_{R}(k, M)=E M,
$$

so the two Morita equivalences consider the left and right adjoints of the same functor.

The unit of the adjunction $M \longrightarrow C E^{\prime}(M)$ is not very well behaved, and the functor $C E^{\prime}$ is not even idempotent in general.

9.C. Complete modules and torsion modules. Even when we are not interested in the intermediate category of $\mathcal{E}$-modules, several of the composite functors give interesting endofunctors of the category of $R$-modules.

LEMMA 9.6. If $k$ is proxy-small then $k$-cellular approximation is smashing:

$$
\operatorname{Cell}_{k} M \simeq\left(\operatorname{Cell}_{k} R\right) \otimes_{R} M .
$$

Proof: Both modules come with natural maps to $M$. Since $R$ builds $M,\left(\operatorname{Cell}_{k} R\right) \otimes_{R}$ $R$ builds $\left(\operatorname{Cell}_{k} R\right) \otimes_{R} M$, so that there is a unique map $\left(\operatorname{Cell}_{k} R\right) \otimes_{R} M \longrightarrow \operatorname{Cell}_{k} M$. 
Assuming $k$ is proxy-small, there is a unique map in the reverse direction, because $\operatorname{Hom}_{R}\left(\operatorname{Cell}_{k} M, \check{C}(k) \otimes_{R} M\right) \simeq 0$. Indeed, this obstruction module cobuilt from $\operatorname{Hom}_{R}\left(K, \check{C}(k) \otimes_{R} M\right)$, and $K^{\#} \otimes_{R} \check{C}(k) \simeq 0$.

We therefore see by 9.3 and 9.6 that if $k$ is proxy-small

$$
\operatorname{Cell}_{k}(M)=T E M=T E^{\prime} M .
$$

This is the composite of two left adjoints, focusing attention on its right adjoint $C E M$, and we note that

$$
C E(M)=\operatorname{Hom}_{R}\left(k^{\#}, \operatorname{Hom}_{R}(k, M)\right)=\operatorname{Hom}_{R}(T E R, M) .
$$

By analogy with Subsection 3.B we may make the following definition.

Definition 9.7. The completion of an $R$-module $M$ is the map

$$
M \longrightarrow \operatorname{Hom}_{R}(T E R, M)=C E M .
$$

We say that $M$ is complete if the completion map is an equivalence.

REMARK 9.8. By 9.6] we see that completion is idempotent.

We adopt the notation

$$
\Gamma_{k} M:=T E^{\prime} M
$$

and

$$
\Lambda^{k} M:=C E M .
$$

This is by analogy with the case of commutative algebra through the approach of Part 1 (see Subsection 3.B), where $\Gamma_{k}=\Gamma_{I}$ is the total right derived functor of the $I$-power torsion functor and $\Lambda^{k}=\Lambda^{I}$ is the total left derived functor of the completion functor (see [1, 2, for the context of commutative rings).

It follows from the adjunctions described in Section 9 that $\Gamma_{k}$ is left adjoint to $\Lambda^{k}$ as endofunctors of the category of $R$-modules:

$$
\operatorname{Hom}_{R}\left(\Gamma_{k} M, N\right)=\operatorname{Hom}_{R}\left(M, \Lambda^{k} N\right)
$$

for $R$-modules $M$ and $N$. Slightly more general is the following observation.

Lemma 9.9. For $R$-modules $M$ and $N$ there is a natural equivalence

$$
\Lambda^{k} \operatorname{Hom}_{R}(M, N) \simeq \operatorname{Hom}_{R}\left(\Gamma_{k} M, \Gamma_{k} N\right) .
$$

Proof: Consider $\operatorname{Hom}_{R}\left(M, \operatorname{Hom}_{R}\left(\Gamma_{k} R, N\right)\right)$. 
Lemma 9.10. If $k$ is proxy-small, $\Gamma_{k}$ and $\Lambda^{k}$ give an adjoint equivalence

$$
\operatorname{Cell}(R, k) \simeq D(\operatorname{comp}-R \text {-mod }),
$$

where $D$ (comp-R-mod) is the triangulated subcategory of $D(R)$ consisting of complete modules.

Proof: We have

$$
T E^{\prime} M \simeq T E M \simeq \Gamma_{k} M \simeq \Gamma_{K} M
$$

and

$$
C E M \simeq \operatorname{Hom}_{R}\left(\Gamma_{k} R, M\right) \simeq \operatorname{Hom}_{R}\left(\Gamma_{K} R, M\right),
$$

so it suffices to prove the result when $k$ is small. When $k$ is small the present adjunction is the composite of two adjoint pairs of equivalences. We have seen this for the first variant, and the second variant is proved similarly by arguing that the unit and counit are equivalences.

\section{Matlis lifts.}

Matlis lifts are the second novel ingredient in the story. They can be viewed as a language for discussing orientations. For a more extensive discussion, see [19].

10.A. The definition. To motivate the definition, suppose $R$ is a $k$-algebra. There are then two dualities on $R$-modules we may wish to consider: the SpanierWhitehead dual

$$
M^{\#}=\operatorname{Hom}_{R}(M, R)
$$

and the Brown-Comenetz dual

$$
M^{\vee}=\operatorname{Hom}_{k}(M, k) .
$$

To see how different these usually are, we may conider the case when $R$ is concentrated in infinitely many positive degrees: then $R^{\#}=R$ is still positively graded and cyclic, whereas $R^{\vee}$ is negatively graded and not finitely generated.

When $R$ is not a $k$-algebra, the situation is a little more complicated, and a guiding example is provided by Pontrjagin duality. For this we consider the case $R=\mathbb{Z}$ and $k=\mathbb{Z} / p$. If $T$ is a $\mathbb{Z} / p$-module, we have the vector space dual $\operatorname{Hom}_{\mathbb{Z} / p}(T, \mathbb{Z} / p)$, but we may wish to extend this to $\mathbb{Z}$-modules, using

$$
\operatorname{Hom}_{\mathbb{Z} / p}(T, \mathbb{Z} / p)=\operatorname{Hom}_{\mathbb{Z}}\left(T, \mathbb{Z} / p^{\infty}\right) ;
$$

we say $\mathbb{Z} / p^{\infty}$ is a Matlis lift of $\mathbb{Z} / p$ from $\mathbb{Z} / p$-modules to $\mathbb{Z}$-modules.

Definition 10.1. Given a ring map $R \longrightarrow k$, and a $k$-module $N$, we say that the $R$-module $I=I(N)$ is a Matlis lift of $N$ if (i) $\operatorname{Hom}_{R}(k, I) \simeq N$ as left $k$-modules and (ii) $I$ is effectively constructible from $k$. 
The first condition is the special case $T=k$ of our motivation, and the general case follows. Since the first condition only sees the $k$-cellularization of $I$, it is natural to require $I$ is $k$-cellular, and the second condition is a small strengthening of this.

It is worth highlighting the fact that the existence of a Matlis lift of $N$ provides a right $\mathcal{E}$-action on $N$ extending its left $k$-module structure. Discussion of Matlis lifts can be reformulated in these terms.

We will only need the special case $N=k$ here. If $k$ is proxy-small, effective constructibility is the same as cellularity so 9.3 shows that Matlis lifts of $k$ correspond to right $\mathcal{E}$-actions on $k$. Given a preferred Matlis lift $I$ of $k$, we write $M^{\vee}=\operatorname{Hom}_{R}(M, I)$ since it extends $k$-duality of $k$-modules.

10.B. Examples. In the particular case of the $k$-module $k$ itself, we may ask how many structures it admits as a right $\mathcal{E}$-module, and for which of these structures a Matlis lift exists. In several interesting cases, there is a unique right action of $\mathcal{E}$ on $k$.

EXAMPLES 10.2. (i) If $R$ is a commutative local ring, the injective hull $I(k)$ is a Matlis lift of $k$. Connectivity arguments show there is a unique right $\mathcal{E}$-action on $k$, and hence $I(k)$ is the unique Matlis lift.

(ii) If $R=C^{*}(X ; k)$, for a connected space $X$, then $\mathcal{E} \simeq C_{*}(\Omega X ; k)$ provided the Eilenberg-Moore spectral sequence converges. Right $\mathcal{E}$-actions on $k$ correspond to actions of $\pi_{0}\left(C_{*}(\Omega X ; k)\right)=k\left[\pi_{1}(X)\right]$ on $k$, which is to say group homomorphisms $\pi_{1}(X) \longrightarrow k^{\times}$. The trivial action is the one specified by the trivial homomorphism, and the associated Matlis lift is $C_{*}(X ; k)$. If $\pi_{1}(X)$ is a $p$-group and $k=\mathbb{F}_{p}$, the homomorphism is necessarily trivial, so $C_{*}(X ; k)$ is the unique Matlis lift of $k$.

(iii) In the equivariant case with $k=F\left(G_{+}, R\right)$, actions of $\mathcal{E}$ on $k$ correspond to actions of $F\left(G_{+}, G_{+}\right)$on $G_{+}$extending the left action of $G_{+}$, and hence there is only one. The Matlis lift of $k$ corresponding to the standard action is $R$ itself.

10.C. Brown-Comenetz duality and Matlis lifts. The p-primary BrownComentez dual $I(p)$ of the sphere spectrum in $p$-local stable homotopy theory is defined by the condition

$$
[T, I(p)]^{n}=\operatorname{Hom}_{\mathbb{Z}}\left(\pi_{n}(T), \mathbb{Q} / \mathbb{Z}_{(p)}\right) .
$$

In fact it is the unique Matlis lift of $H \mathbb{F}_{p}$ along $R=S^{0} \longrightarrow H \mathbb{F}_{p}=k$ and

$$
I(p)=H \mathbb{F}_{p} \otimes_{\mathcal{E}} H \mathbb{F}_{p},
$$

where $\mathcal{E}=\operatorname{End}_{S^{0}}\left(H \mathbb{F}_{p}\right)$ is the mod $p$ Steenrod algebra ring spectrum. 
Proof: To see that $I(p)$ is a Matlis lift, we note that by definition

$$
\operatorname{Hom}_{S^{0}}\left(H \mathbb{F}_{p}, I(p)\right)=H \mathbb{F}_{p} .
$$

By connectivity of $\mathcal{E}$, there is a unique right $\mathcal{E}$-action on $H \mathbb{F}_{p}$.

Since $H \mathbb{F}_{p}$ is not proxy-small, we must argue directly that $I(p)$ is effectively constructible. The counit of the adjunction gives a comparison map $H \mathbb{F}_{p} \otimes_{\mathcal{E}} H \mathbb{F}_{p} \longrightarrow$ $I(p)$. The homotopy groups of both sides are calculated by a spectral sequence with $E^{2}$-term $\operatorname{Tor}_{i}^{\mathcal{A}_{p}}\left(\mathbb{F}_{p}, \mathbb{F}_{p}\right)$, on the left it is a bar spectral sequence and on the right a dual Adams spectral sequence. In fact we may dualize the usual construction of the Adams spectral sequence and observe it also gives a calculation of the bar spectral sequence, so the map induces an isomorphism of $E^{2}$-terms.

10.D. Local duality and the dualizing complex. A dualizing complex $D$ for a commutative Noetherian local ring $R$ is characterized by the three requirements (i) $\operatorname{Hom}_{R}(D, D)=R$, (ii) $D$ has finite injective dimension and (iii) $H_{*}(D)$ is a finitely generated $R$-module. There is a useful summary of properties in [5].

Dualizing complexes exist under very weak hypotheses, certainly if the ring is a quotient of a complete local ring. We only use the fact that for a local ring $R$ the dualizing complex $D$ has the property that $\Gamma_{k} D=\mathbb{I}(k)$ (up to suspension). From the fact that $\Gamma_{k}$ and $\Lambda^{k}$ give inverse equivalences, it is therefore natural to make the following definition.

Definition 10.3. If there is a Matlis lift $\mathbb{I}(k)$ of $k$, we define the dualizing complex by

$$
D=\Lambda^{k} \mathbb{I}(k) .
$$

REMARK 10.4. To justify calling $D$ the dualizing complex, we should establish a natural equivalence

$$
R \longrightarrow \operatorname{Hom}_{R}(D, D)=\operatorname{Hom}_{R}(\mathbb{I}(k), \mathbb{I}(k)),
$$

in some generality, at least when $R$ is complete. Here are two examples from local algebra.

(a) Suppose $R$ is a $k$-algebra. In this case $\operatorname{Hom}_{R}(\mathbb{I}(k), \mathbb{I}(k))=\operatorname{Hom}_{k}(\mathbb{I}(k), k)$. If $R$ is $k$-reflexive (for example if $k$ is a field and $H_{*}(R)$ is bounded below and a finite dimensional vector space in each degree) we also have $\mathbb{I}(k)=\operatorname{Hom}_{k}(R, k)$, and the required equivalence follows.

(b) Suppose $R$ is a complete, commutative local ring. Suppose also that there is a map $Q \longrightarrow R$ making $R$ into a finite $Q$-module and with $Q$ complete and Gorenstein. 
In that case $\Gamma_{I Q}=\Gamma_{I}$ on $R$-modules, and $\Gamma_{I} Q=\mathbb{I}_{k}^{Q}(k)$ is the Matlis lift of $k$ to a $Q$-module. Hence

$$
Q=\operatorname{Hom}_{Q}\left(\Gamma_{I} Q, \Gamma_{I} Q\right)=\operatorname{Hom}_{Q}\left(\mathbb{I}_{k}^{Q}(k), \mathbb{I}_{k}^{Q}(k)\right) .
$$

We now claim

$$
\mathbb{I}_{k}^{R}(k):=\operatorname{Hom}_{Q}\left(R, \mathbb{I}_{k}^{Q}(k)\right)
$$

is a Matlis lift of $k$ to an $R$-module. We may then calculate

$$
R=Q \otimes_{Q} R=\operatorname{Hom}_{Q}\left(\mathbb{I}_{k}^{Q}(k), \mathbb{I}_{k}^{Q}(k)\right) \otimes_{Q} R=\operatorname{Hom}_{R}\left(\mathbb{I}_{k}^{R}(k), \mathbb{I}_{k}^{R}(k)\right)
$$

We may connect the dualizing complex with local duality by a tautology.

COROLlaRY 10.5. (Local duality) If $\mathbb{I}(k)$ is a Matlis lift of $k$ there is an equivalence

$$
\operatorname{Hom}_{R}\left(\Gamma_{k} R, \mathbb{I}(k)\right)=\Lambda^{k} D
$$

\section{The Gorenstein condition.}

A commutative Noetherian local ring $R$ is Gorenstein if and only if $\operatorname{Ext}_{R}^{*}(k, R)$ is one dimensional as a $k$-vector space [48, 18.1]. In the derived category we can restate this as saying that the homology of the (right derived) Hom complex $\operatorname{Hom}_{R}(k, R)$ is equivalent to a suspension of $k(\mathrm{cf}[\mathbf{2 6}]$ ). This suggests the definition for ring spectra.

Definition 11.1. 19. We say that $R \longrightarrow k$ is Gorenstein if there is an equivalence of $R$-modules $\operatorname{Hom}_{R}(k, R) \simeq \Sigma^{a} k$ for some integer $a$.

As in commutative algebra, Gorenstein rings are ubiquitous.

EXAMPLES 11.2. (i) The ring spectrum associated to a commutative local ring $R$ is Gorenstein if and only if the local ring is Gorenstein in the conventional sense, as we discussed in our motivation.

(ii) The ring spectrum $R=C^{*}(X ; k)$ is Gorenstein in two cases. First there is the more familiar case when $X$ is a compact connected manifold orientable over $k$. In fact $R$ is also Gorenstein if $X$ is not orientable, provided $2^{n}$ acts as zero on $k$ for some $n$. To see this, let $\tilde{R}=C^{*}(\tilde{X} ; k)$ denote the cochain complex of the orientable double cover $\tilde{X}$ of $X$, and let $C$ be the group of order 2, acting via covering transformations. Notice that the convergence of the Rothenberg-Steenrod and Eilenberg-Moore spectral sequences give $\operatorname{Hom}_{k C}(k, \tilde{R}) \simeq R$ and $\tilde{R} \otimes_{R} k \simeq k C$. 
Now we may calculate

$$
\begin{aligned}
\operatorname{Hom}_{R}(k, R) & \simeq \operatorname{Hom}_{R}\left(k, \operatorname{Hom}_{k C}(k, \tilde{R})\right) \\
& \simeq \operatorname{Hom}_{R}\left(k \otimes_{k C} k, \tilde{R}\right) \\
& \simeq \operatorname{Hom}_{\tilde{R}}\left(\tilde{R} \otimes_{R} k \otimes_{k C} k, \tilde{R}\right) \\
& \simeq \operatorname{Hom}_{\tilde{R}}\left(k C \otimes_{k C} k, \tilde{R}\right) \\
& \simeq \operatorname{Hom}_{\tilde{R}}(k, \tilde{R}) .
\end{aligned}
$$

Secondly, $R$ is Gorenstein when $X=B G$ for any finite group $G$. We will give the proof when $G$ is a finite $p$-group in Subsection 11.D below. In fact this example extends to many cases when $G$ is a compact Lie group. To explain, we need to consider the adjoint action of $\pi_{1}(B G)$ on $H_{c}^{d}\left(T_{e} G ; k\right) \cong k$, where $G$ is of dimension $d$. The ring $R$ is Gorenstein if the action is trivial or if $2^{n}$ acts as 0 on $k$.

(iii) The group ring $k G$ of a finite group $G$ is Gorenstein as a ring spectrum. More generally, if $R$ is an equivariant commutative ring spectrum and $k=F\left(G_{+}, R\right)$ represents the nonequivariant theory, then

$$
\operatorname{Hom}_{R}(k, R)=\operatorname{Hom}_{R}\left(F\left(G_{+}, R\right), R\right) \simeq G_{+} \wedge \operatorname{Hom}_{R}(R, R) \simeq k,
$$

so that $R$ is Gorenstein.

11.A. Orientability. For simplicity we suppose for the rest of the section that all cellular objects are effectively constructible, for example if $k$ is proxy-small.

If $R$ is Gorenstein, $k$ acquires new structure: that of a right $\mathcal{E}$-module (not to be confused with its natural structure as a left $\mathcal{E}$-module), and $\Gamma_{k} R$ is a Matlis lift of $k$. We want to say that $R$ is orientable if the new right action is 'trivial'. In many examples there is a natural candidate for an action to be called trivial, and a corresponding Matlis lift $I$ of $k$.

Definition 11.3. A Gorenstein ring spectrum $R$ is orientable if

$$
\operatorname{Hom}_{R}(k, R) \simeq \Sigma^{a} \operatorname{Hom}_{R}(k, I)
$$

as right $\mathcal{E}$-modules.

ExAmPles 11.4. (i) If $R$ is a commutative local ring, we remarked in Example 10.2 that there is a unique right $\mathcal{E}$-module structure on $k$, and hence a unique Matlis lift of $k$. Thus every Gorenstein commutative ring is orientable as a ring spectrum. The notion of orientability is redundant in classical commutative algebra.

(ii) If $R=C^{*}(X ; k)$, we have already remarked that the Matlis lift corresponding to the trivial action is $I=C_{*}(X ; k)$.

If $X$ is a manifold for which $R=C^{*}(X ; k)$ is Gorenstein, the associated action is the one arising from the monodromy action. Thus $R$ is orientable if and only if the manifold $X$ is orientable over $k$. For example if $X$ is not orientable and $k=\mathbb{Z} / 4$, the ring spectrum $R$ is Gorenstein but not orientable. 
Similarly, if $X=B G$, the Gorenstein action is the adjoint action of $\pi_{1}(B G)$ on $H_{c}^{d}\left(T_{e} G ; k\right)$, where $G$ is of dimension $d$. The case $G=O(2)$ gives an example where this is non-trivial, since the reflections act as -1 . The ring $k=\mathbb{Z} / 4$ is still proxy-small so $R$ is Gorenstein but not orientable.

(iii) In the equivariant example with $k=F\left(G_{+}, R\right)$, there is an obvious action of $F\left(G_{+}, G_{+}\right)$on $G_{+}$, giving an action of $\mathcal{E}$ on $k$, which we use to give the Matlis lift. The Gorenstein action is its opposite; since the two are isomorphic, this example is orientably Gorenstein.

11.B. The local cohomology theorem. We saw in 5.3 that the stable Koszul complex provides a construction of $k$-cellular approximation in many contexts. In this case, its homotopy is calculated using local cohomology: there is a spectral sequence

$$
H_{\mathfrak{m}}^{*}\left(\pi_{*}(R)\right) \Rightarrow \pi_{*}\left(\Gamma_{k} R\right) .
$$

We can then deduce a valuable duality property from the Gorenstein condition. Indeed, if $R \longrightarrow k$ is Gorenstein and orientable, we have the equivalences

$$
E \Gamma_{k} R=\operatorname{Hom}_{R}\left(k, \Gamma_{k} R\right) \simeq \operatorname{Hom}_{R}(k, R) \simeq \Sigma^{a} \operatorname{Hom}_{R}(k, I)=E \Sigma^{a} I
$$

of right $\mathcal{E}$-modules. By 9.3 we conclude

$$
\Gamma_{k} R \simeq T E \Gamma_{k} R \simeq T E \Sigma^{a} I \simeq \Sigma^{a} I .
$$

For example if $R$ is a $k$-algebra for a field $k$, we can take $I=\operatorname{Hom}_{k}(R, k)$ provided it is $k$-cellular, and conclude that there is a spectral sequence

$$
H_{\mathfrak{m}}^{*}\left(\pi_{*}(R)\right) \Rightarrow H^{*}\left(\operatorname{Hom}_{k}(R, k)\right)=\operatorname{Hom}_{k}\left(\pi_{*} R, k\right) .
$$

In particular if $\pi_{*}(R)$ is Cohen-Macaulay, this spectral sequence collapses to show it is also Gorenstein. In fact one may apply Grothendieck's dual localization process to this spectral sequence and hence conclude that whatever its depth, $\pi_{*}(R)$ is generically Gorenstein 33. This dual localization process is lifted to the level of module spectra in $\mathbf{9}$.

EXAMPLES 11.5. (i) When $R$ is a commutative local ring, we just obtain Gorenstein duality, stating that a Gorenstein ring is Cohen-Macaulay and $H_{\mathfrak{m}}^{r}(R)=I(k)$.

(ii) If $R=C^{*}(X ; k)$ for an orientable manifold $X$ we deduce the Poincaré duality statement

$$
H^{*}(X ; k)=H_{\mathfrak{m}}^{0}\left(H^{*}(X ; k)\right) \cong H_{*}(X ; k) .
$$

A much more interesting example is that of $C^{*}(B G)$ for a compact Lie group $G$. Since $\pi_{*}\left(C^{*}(B G)\right)=H^{*}(B G)$, when $G$ is finite there is a spectral sequence

$$
H_{\mathfrak{m}}^{*}\left(H^{*}(B G)\right) \Rightarrow H_{*}(B G),
$$


showing that the group cohomology ring $H^{*}(B G)$ has very special properties, such as being generically Gorenstein.

(iii) For the group ring, we conclude $k G=H_{\mathfrak{m}}^{0}(k G)=k G^{\vee}$, which is to say that $k G$ is a Frobenius algebra.

For the general equivariant example, with $k \simeq F\left(G_{+}, R\right)$, we have $\Gamma_{k} R \simeq$ $R \wedge E G_{+}$. However, in this case $\operatorname{Hom}_{k}(R, k) \simeq R$, which is not $k$-cellular: the statement $\Gamma_{k} R \simeq \Gamma_{k}\left(R^{\vee}\right)$ is a tautology. The only content in this case comes from the work of Section 17 of Part 1, where we say $R \wedge E G_{+} \simeq \Gamma_{k} R \simeq \Gamma_{I} R$, converting the geometric cellular approximation to an algebraic one.

11.C. The Gorenstein condition and the dualizing complex. Another characterization of the Gorenstein condition for complete local rings is condition on $R$ that $R \simeq \Sigma^{a} D$ for some $a$, where $D$ is the dualizing complex. Combining this with the tautologous local duality statement 10.5 we see that this version of the Gorenstein condition implies

$$
\operatorname{Hom}_{R}\left(\Gamma_{k} R, \mathbb{I}(k)\right)=\Sigma^{a} R .
$$

Example 11.6. The example of Mahowald-Rezk 47 fits well here. They define the $n$th dualizing complex by

$$
W_{n} S^{0}=I C_{n}^{f} S^{0}
$$

where $I$ denotes Brown-Comenetz duality, and $C_{n}^{f}$ is the finite counterpart of the $n$th acyclization discussed in Section [6] namely the $F(n+1)$-cellular approximation functor, for a finite type $n$-spectrum $F(n+1)$. Thus it is natural to write $C_{n}^{f} S^{0}=$ $\Gamma_{F(n+1)} S^{0}$, and hence its Brown-Comenetz dual is the dualizing complex.

Mahowald and Rezk consider the class of spectra for which their cohomology is finitely presented over the Steenrod algebra. They prove that all such type $n \mathrm{fp}$ spectra are reflexive. In fact they go on to form $W X$ as a direct limit of $W_{n} X$ and show that for many interesting fp ring spectra (such as $X=k u, k o, e o_{2}, B P\langle n\rangle$ ) we have $W X \simeq \Sigma^{d} X$ for a suitable $d$. This says that the dualizing complex is a suspension of the ring, and we have just observed this is a form of the Gorenstein condition.

11.D. Morita invariance of the Gorenstein condition. In this final section we show that the Gorenstein condition is Morita invariant in many useful cases, provided $R$ is a $k$-algebra. This allows us to deduce striking consequences from well-known examples of Gorenstein rings. For instance we can deduce the local cohomology theorem for finite $p$-groups from the fact that $k G$ is a Frobenius algebra. 
TheOrem 11.7. Suppose $R$ is a $k$-algebra, and $R^{\vee}$ is $k$-cellular and finally that $\mathcal{E}$ and $R$ are Matlis reflexive. Then

$$
\operatorname{Hom}_{\mathcal{E}}(k, \mathcal{E}) \cong \operatorname{Hom}_{R}(k, R)
$$

and hence

$$
\mathcal{E} \text { is Gorenstein } \Longleftrightarrow R \text { is Gorenstein. }
$$

Proof: We use the fact that $E\left(R^{\vee}\right)=k^{\vee}$, so that we have

$$
R^{\vee}=T E\left(R^{\vee}\right)=T k^{\vee}=k^{\vee} \otimes_{\mathcal{E}} k,
$$

and the fact that $\mathcal{E}=\operatorname{Hom}_{R}(k, k)$ dualizes to give

$$
\mathcal{E}^{\vee}=k \otimes_{R} k^{\vee} .
$$

Next, note that the expression $k \otimes_{R} k^{\vee} \otimes_{\mathcal{E}} k$ makes sense, where the right $\mathcal{E}$ module structure on the first two factors comes from $k^{\vee}$. The key equality in the proof is simply the associativity isomorphism

$$
\mathcal{E}^{\vee} \otimes_{\mathcal{E}} k=k \otimes_{R} k^{\vee} \otimes_{\mathcal{E}} k=k \otimes_{R} R^{\vee} .
$$

Now we make the following calculation,

$$
\begin{aligned}
\operatorname{Hom}_{\mathcal{E}}(k, \mathcal{E}) & \simeq \operatorname{Hom}_{\mathcal{E}}\left(k,\left(\mathcal{E}^{\vee}\right)^{\vee}\right) \\
& \simeq \operatorname{Hom}_{k}\left(\mathcal{E}^{\vee} \otimes_{\mathcal{E}} k, k\right) \\
& \simeq \operatorname{Hom}_{k}\left(k \otimes_{R} k^{\vee} \otimes_{\mathcal{E}} k, k\right) \\
& \simeq \operatorname{Hom}_{k}\left(k \otimes_{R} R^{\vee}, k\right) \\
& \simeq \operatorname{Hom}_{R}\left(k,\left(R^{\vee}\right)^{\vee}\right) \\
& \simeq \operatorname{Hom}_{R}(k, R)
\end{aligned}
$$

Corollary 11.8. If $G$ is a finite p-group then $k G$ is Gorenstein and hence $C^{*}(B G)$ is Gorenstein.

\section{References}

[1] L.Alonso Tarrio, A.Jeremias Lopez and J. Lipman "Local homology and cohomology on schemes." Ann.Scient. Ec.Norm.Sup. 30 (1997) 1-39

[2] L.Alonso Tarrio, A.Jeremias Lopez and J. Lipman "Studies in duality on Noetherian formal schemes and non-Noetherian ordinary schemes" Cont. Math. 244 AMS (1999) x+126

[3] M.F.Atiyah and G.B. Segal "Equivariant $K$-theory and completion" J. Differential Geometry $319691-18$

[4] L.L. Avramov and H.-B. Foxby "Locally Gorenstein homomorphisms." American J. Math. 114 (1992) 1007-1047

[5] L.L. Avramov and H.-B. Foxby "Ring homomorphism and finite Gorenstein dimension" PLMS 75 (1997) 241-270

[6] H.Bass "On the ubiquity of Gorenstein rings" Math. Z. 821963 8-28.

[7] H.Bass "Algebraic K-theory." Benjamin (1968) xx+762pp 
[8] D.J.Benson and J.P.C.Greenlees "Commutative algebra for cohomology rings of classifying spaces of compact Lie groups." JPAA 122 (1997) 41-53

[9] D.J.Benson and J.P.C.Greenlees "Duality in topology and modular representation theory." Preprint (2004)

[10] A.K. Bousfield. The localization of spaces with respect to homology. Topology 14 (1975), 133-150.

[11] A.K. Bousfield. The localization of spectra with respect to homology. Topology 18 (1979), 257-281.

[12] A.K. Bousfield and D.M. Kan. Homotopy limits, completions and localizations. Springer Lecture notes in mathematics Vol. 304. 1972.

[13] W.Bruns and Herzog "Cohen-Macaulay rings." Cambridge Studies in Advanced Mathematics, 39. Cambridge University Press, Cambridge, 1993. xii+403 pp.

[14] G. Carlsson "Equivariant stable homotopy and Segal's Burnside ring conjecture." Ann. of Math. (2) 120 (1984), no. 2, 189-224.

[15] E. Devinatz. Small ring spectra. J. Pure and Applied Algebra 81 (1992), 11 -16.

[16] E. Devinatz, M.J. Hopkins and J. Smith. Nilpotence and stable homotopy theory. Annals of Maths. 128 (1988), 207-242.

[17] W.G.Dwyer "Strong convergence of the Eilenberg-Moore spectral sequence" Topology 13 (1974) 255-265

[18] W.G.Dwyer and J.P.C.Greenlees "Complete modules and torsion modules" American J. Math. 124 (2002) 199-220

[19] W.G.Dwyer, J.P.C.Greenlees and S.B.Iyengar "Duality in algebra and topology." Advances in Maths 200 (2006) 357-402

[20] W.G.Dwyer and J.P.C.Greenlees and S.B.Iyengar "Finiteness conditions in derived categories of local rings." Comm. Math. Helv. 81 (2006) 383-432

[21] W.G.Dwyer and J.P.C.Greenlees and S.B.Iyengar "Dualities in chromatic stable homotopy theory." (in preparation)

[22] W.G.Dwyer and C.Wilkerson "Homotopy fixed-point methods for Lie groups and finite loop spaces" Ann. Math. 139 (1994) 395-442

[23] A.D. Elmendorf, J.P.C. Greenlees, I. Kriz and J.P. May. Commutative algebra in stable homotopy theory and a completion theorem. Math. Res. Letters 1 (1994) 225-239.

[24] A. D. Elmendorf, I. Kriz, M. A. Mandell, and J. P. May. Rings, Modules and Algebras in Stable Homotopy Theory, Volume 47 of Amer. Math. Soc. Surveys and Monographs. American Mathematical Society, 1996.

[25] A. D. Elmendorf, and J. P. May "Algebras over equivariant sphere spectra" J. Pure and Applied Algebra 116 (1997) 139-149

[26] Y. Félix, S. Halperin and J.-C. Thomas "Gorenstein spaces" Advances in Mathematics, 71, (1988) 92-112

[27] J.P.C.Greenlees "K-homology of universal spaces and local cohomology of the representation ring" Topology 32 (1993) 295-308.

[28] J.P.C.Greenlees "Tate cohomology in commutative algebra." J. Pure Appl. Algebra 94 (1994), no. 1, 59-83.

[29] J.P.C.Greenlees "Commutative algebra for group cohomology." JPAA 98 (1995) 151-162

[30] J.P.C.Greenlees "Tate cohomology in axiomatic stable homotopy theory." Cohomological methods in homotopy theory (Bellaterra, 1998), 149-176, Progr. Math., 196, Birkhuser, Basel, 2001.

[31] J.P.C.Greenlees "Local cohomology in equivariant topology." Local cohomology and its applications (Guanajuato, 1999), 1-38, Lecture Notes in Pure and Appl. Math., 226, Dekker, New York, 2002.

[32] J.P.C.Greenlees "Spectra for commutative algebraists." Proceedings of the Chicago conference, This volume, 25pp.

[33] J.P.C.Greenlees and G.Lyubeznik "Rings with a local cohomology theorem with applications to cohomology rings of groups." JPAA 149 (2000) 267-285

[34] J.P.C. Greenlees and J.P. May. Completions of G-spectra at ideals of the Burnside ring. Proc. Adams Memorial Symposium, Volume II, CUP (1992), 145-178.

[35] J.P.C. Greenlees and J.P. May. Derived functors of I-adic completion and local homology. J. Algebra 149 (1992), 438-453. 
[36] J.P.C.Greenlees and J.P. May "Equivariant stable homotopy theory". Handbook of algebraic topology, 277-323, North-Holland, Amsterdam, 1995.

[37] J.P.C.Greenlees and J.P. May "Completions in algebra and topology." Handbook of algebraic topology, 255-276, North-Holland, Amsterdam, 1995.

[38] J.P.C.Greenlees and J.P.May "Localization and completion theorems for $M U$-module spectra." Annals of Maths. 146 (1997) 509-544

[39] J.P.C. Greenlees and J.P. May. Generalized Tate cohomology. Mem. American Math. Soc. 1994 (to appear).

[40] A. Grothendieck. Sur quelques points d'algèbre homologique. Tohoku Mathematical Journal 9 (1957), 119-221.

[41] A. Grothendieck (notes by R. Hartshorne). Local cohomology. Springer Lecture notes in mathematics Vol. 41. 1967.

[42] R. Hartshorne. Algebraic Geometry. Springer-Verlag. 1977.

[43] M.J. Hopkins. Global methods in homotopy theory. Proc. of the 1985 LMS Symposium on Homotopy Theory. London Math. Soc. 1987, 73-96.

[44] M.J. Hopkins and J.H. Smith. "Nilpotence and stable homotopy theory. II." Ann. of Math. (2) 148 (1998), no. 1, 1-49.

[45] M.Hovey and N.P.Strickland "Morava K-theories and localisation." Mem. AMS 139 (1999) viii+100pp

[46] R. Levi "On homological rate of growth and the homotopy type of $\Omega\left(B G_{p}^{\wedge}\right)$." Math. Z. 226 (1997) 429-444

[47] M.E.Mahowald and C. Rezk "Brown-Comenetz duality and the Adams spectral sequence" AJM 121 (1999) 1153-1177

[48] H. Matsumura. Commutative ring theory. Cambridge Univ. Press. 1986.

[49] D.C. Ravenel. Localization with respect to certain periodic homology theories. American J. Math. 106 (1984), 351-414.

[50] D.C. Ravenel. The geometric realization of the chromatic resolution. In Algebraic topology and algebraic $K$-theory. Princeton University Press, 1987, 168-179.

[51] D.C. Ravenel. Nilpotence and periodicity in stable homotopy theory. Princeton University Press. 1992.

[52] P. Schenzel "Proregular sequences, local cohomology, and completion." Math. Scand. 92 (2003), no. 2, 161-180.

[53] S. Schwede, "Morita theory in abelian, derived and stable model categories." Structured ring spectra, 33-86, London Math. Soc. Lecture Note Ser., 315, Cambridge Univ. Press, Cambridge, 2004.

[54] B.E.Shipley " $H \mathbb{Z}$-algebra spectra are differential graded algebras." Preprint 14pp, (available at http://www.math.purdue.edu/ bshipley//

[55] D. Sullivan. The genetics of homotopy theory and the Adams conjecture. Annals of Math. 100 (1974), 1-80.

The University of Sheffield, Sheffield, S3 7RH UK

E-mail address: j.greenlees@sheffield.ac.uk 